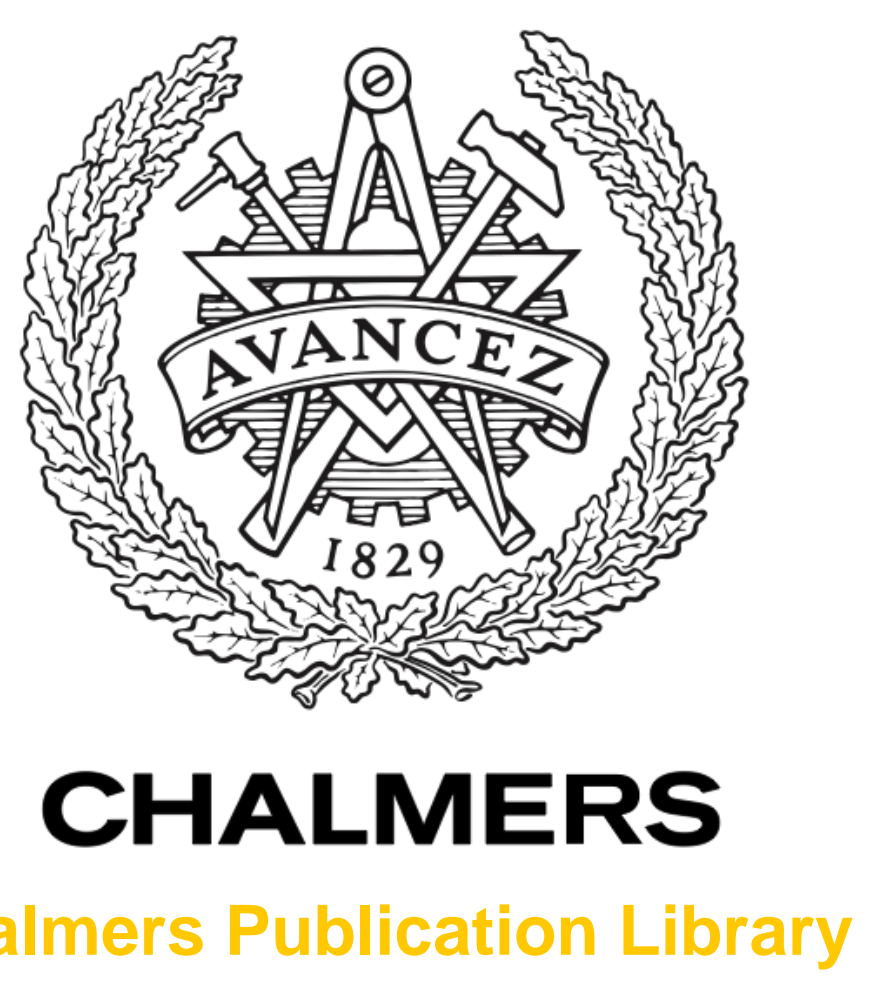

Chalmers Publication Library

\title{
Joint Time-Frequency Analysis of Transient Electromagnetic Scattering from a Subsurface Target
}

This document has been downloaded from Chalmers Publication Library (CPL). It is the author's version of a work that was accepted for publication in:

IEEE Antennas and Propagations Magazine (ISSN: 1045-9243)

Citation for the published paper:

Lui, H. ; Shuley, N. ; Persson, M. (2012) "Joint Time-Frequency Analysis of Transient Electromagnetic Scattering from a Subsurface Target". IEEE Antennas and Propagations

Magazine, vol. 54(5), pp. 109-130.

http://dx.doi.org/10.1109/MAP.2012.6348122

Downloaded from: http://publications.lib.chalmers.se/publication/157341

Notice: Changes introduced as a result of publishing processes such as copy-editing and formatting may not be reflected in this document. For a definitive version of this work, please refer to the published source. Please note that access to the published version might require a subscription.

Chalmers Publication Library (CPL) offers the possibility of retrieving research publications produced at Chalmers University of Technology. It covers all types of publications: articles, dissertations, licentiate theses, masters theses, conference papers, reports etc. Since 2006 it is the official tool for Chalmers official publication statistics. To ensure that Chalmers research results are disseminated as widely as possible, an Open Access Policy has been adopted.

The CPL service is administrated and maintained by Chalmers Library. 


\title{
Joint Time-Frequency Analysis of Transient Electromagnetic Scattering from a Subsurface Target
}

\author{
Hoi-Shun Lui', Mikael Persson', and Nicholas V. Z. Shuley ${ }^{2}$ \\ ${ }^{1}$ Department of Signals and Systems \\ Chalmers University of Technology \\ SE-412 96 Gothenburg, Sweden \\ E-mail: antony.lui@chalmers.se; lui@ieee.org \\ ${ }^{2}$ School of Information Technology and Electrical Engineering \\ The University of Queensland \\ Brisbane 4072, Australia \\ E-mail: shuley@itee.uq.edu.au
}

\begin{abstract}
The complexity of transient electromagnetic scattering from objects in free space has been well known for years. Such complexity is mainly due to the high aspect dependency of the early-time response, and the interactions between the various scattering events. Attempts have been made to analytically and numerically study the transient scattering problem using time-frequency (TF) analysis. Using this technique, the occurrences of the scattering events and their frequency content can be revealed, which leads to further information about the actual scattering behavior. For the case of subsurface targets, the entire problem becomes more complicated with the introduction of a dielectric interface. Analytical solutions of such complicated electromagnetic problems are usually not available, and can only be numerically addressed in most cases. In this paper, a systematic study of transient scattering from some subsurface targets is presented. The various interactions between the target and the interface of the half-space can be clearly observed. The findings from this work have opened up some issues that are left for further investigation.
\end{abstract}

Keywords: Time domain electromagnetics; transient scattering; subsurface target detection; ultra wideband radar; radar target recognition; time-frequency analysis; electromagnetic scattering

\section{Introduction}

$\mathbf{U}$ ltra-wideband (UWB) transient electromagnetic scattering from radar targets has been a well-researched topic over the years. One well-known application is automatic target recognition (ATR), based on the target-dependent natural resonances embedded in the transient electromagnetic signature [1-3]. According to the Singularity Expansion Method (SEM), the late-time response of the transient scattering from a perfectly electrically conducting (PEC) target can be written as a sum of damped exponentials [3, 4]. Theoretically, these natural resonant frequencies correspond purely to target features, which can be used for target characterization. Resonancebased radar target recognition using K-pulse [5], E-pulse [1, $2,6]$ and S-pulse $[1,2]$ algorithms have been studied as far back as the 1980s. The concept of using target resonances for automatic target recognition was first applied to radar targets in free space, and then later extended to both PEC and dielectric targets sited below a dielectric half-space (a subsurface target) [7-10]. Recent attempts by Lui and Shuley et al. [11-13] have looked into the possibility of using the E-pulse technique for subsurface target detection and recognition.

In addition to the various applications of the original Singularity Expansion Method model for target recognition, another research interest is the theoretical development of the Singularity Expansion Method, especially for the complicated early-time target response. Such complexity is due to the fact that the target has not been fully excited in the early time period, and thus the early-time response is highly dependent 
on the incident aspect, which makes it difficult to model [1420]. Felsen, Heyman, and Shirai [16-20] conducted a number of analytical studies of transient electromagnetic scattering, and proposed the Hybrid Wavefront Singularity Expansion Method (HWSEM). The Hybrid Wavefront Singularity Expansion Method aims to unify the early-time local wavefront-scattering phenomena and the late-time global resonance behavior. It also provides further physical insight into the transient scattering problem. Most analytical studies of transient scattering have been limited to canonical structures, for good reasons. With the rapid development of numerical modeling techniques and measurement facilities in recent years, the target signature of complex targets (non-canonical) can be easily obtained. However, it is difficult to gain any physical insight into the actual scattering mechanisms from computed or measured responses.

One possible approach to assist in the development of better algorithms for subsurface scattering is to study the timevariant transient response in a signal-processing context using joint time-frequency (TF) analysis. In the joint time-frequency domain, the simultaneous existence and occurrence of the frequency components and their temporal development can easily be determined, allowing observation of the establishment of the resonances and their various interactions. There have been a number of studies using time-frequency analysis to study transient electromagnetic scattering for objects in free space (for example, [21-28]). However, most work did not relate the observations from the time-frequency plots to the actual scattering mechanisms. An exception was the work of Rothwell et al. [29], where adaptive time-frequency analysis was used to study the substructure resonances of a model aircraft. Recently, Lui and Shuley [30] applied time-frequency analysis to study scattering from a bent-wire target, and verified the correctness of the Hybrid Wavefront Singularity Expansion Method model. For subsurface targets, there are only a few studies in the literature $[31,32]$. To our knowledge, there has so far not been any work that uses time-frequency analysis for transientscattering phenomena from a subsurface target.

Therefore, the main objective in this work is to study transient electromagnetic scattering with particular emphasis on the scattering events and mechanisms using time-frequency analysis. In particular, examples of wire targets in lossless and lossy subsurface environments are considered. The wire target was chosen because of ease of computation, and its associated high $Q$ allows resonance behavior to be easily identified. Subsequently, results for a hip prosthesis model that has a similar length as the wire but with a lower $Q$ factor will be presented [11-13]. Before considering these examples, a short review of transient electromagnetic scattering of subsurface targets and time-frequency analysis in the context of transient electromagnetic scattering is first given. The findings from the numerical examples and their connections with some recent developments in the automatic target recognition context are discussed in Section 5. Conclusions are reached at the end of the paper.

\section{Transient Electromagnetic Scattering of Subsurface Targets}

Transient electromagnetic scattering of targets embedded within a lossy homogenous half-space have been addressed in a number of papers [33-35]. Compared to transient scattering for objects in free space, the electromagnetic scattering problem for a subsurface target becomes much more complicated, due to the existence of the dielectric discontinuity of the halfspace. Studies using full-wave electromagnetic modeling have shown that there exist two types of resonances: the "image resonance," and the "self resonance" [7, 8]. The "image resonance" corresponds to the interaction between the target and the dielectric interface. This particular resonance varies as the depth of the target changes. Just as for the target in free space, the "self resonance" mainly corresponds to the resonant behavior of the target itself within the dielectric environment. However, compared to a target in free space, which is independent of excitation aspect, the "self resonance" for a subsurface target does slightly vary as a function of target depth, of target orientation, and of the dielectric properties of the surrounding environment $[7,8]$. As a result, subsurface target recognition based on the resonant frequency itself is a complicated process, unless the target depth and orientation are known a priori, such that the exact value of the target resonance is known.

\section{Time-Frequency Analysis in the Context of Transient Electromagnetic Scattering}

The motivation for time-frequency analysis originates from the nature of non-stationary signals, where the frequency components of the signals are time variant. According to the nature of the time-frequency distributions (TFDs), they can be divided into two main classes: linear and bilinear. To further improve the performance of time-frequency distributions, techniques such as time-scaled distributions (TSDs) [36] and reassignment methods [37] have been introduced. It is to be noted that extensive work has been done on time-frequency distributions to ascertain whether those distributions maintain certain desirable properties (e.g., real valued, preserved time and frequency shift, time and frequency margins, instantaneous frequency, group delay, non-negativity, etc.) [38-40]. In this paper, we focus on applying the time-frequency distributions to study the temporal occurrence and frequency of the scattering phenomena of the radar targets. Reviews of various timefrequency distributions can easily be found in [38-41], and thus we are not going to repeat that here. Although time-frequency distributions such as the Choi-William Distribution (CWD) may result in better time-frequency resolution, they can at the same time also introduce other artifacts, which can be misinterpreted. With our previous experience with targets in free space [30], the spectrogram (SP) and smooth-pseudo Wigner-Ville distribution (SPWVD) will be used in this work. For the rest of this section, 
a brief review of time-frequency analysis as applied to transient electromagnetic scattering will be given.

Ling et al. [21, 22] first applied the spectrogram and continuous Wavelet Transform (CWT) to various electromagnetic scattering applications. These included a plasma cylinder [22], an open-ended waveguide cavity [24], a dielectric grating [25], and coated dielectric wires [26]. The frequency of interest was in the quasi-optical region, and the objective was to study the high-frequency scattering mechanisms, such as edge and corner diffractions, most of which can be described by the Uniform Theory of Diffraction (UTD) in the electromagnetic context. Most of Ling's work was concerned with time-frequency analysis of high-frequency electromagnetic scattering and its application to radar imaging. There has not been much work concerning time-frequency analysis in the area of ultrawideband transient scattering. A summary of such work may be found in [21] and [27].

Gaunaurd and Strifors et al. [42] have applied the shorttime Fourier transform (STFT) and the pseudo-Wigner-Ville distribution (PWVD) to impulse-radar applications. They have looked into the target response for some scale-model aircraft and spheres in free space, under impulse excitation with a bandwidth of $2 \mathrm{GHz}$. They found that time-frequency analysis is capable of revealing some of the scattering mechanisms, such as specular returns, creeping waves, and resonances, in the time-frequency plane. They also conducted a brief study using other time-frequency distributions, such as the Choi-William distribution, the Zhao-Atlas-Marks distribution (ZAMD), the Gabor spectrogram, and the adaptive spectrogram [42] on the same target response. They concluded that the pseudo-WignerVille distribution and Choi-William distribution are to be preferred, with good capabilities of cross-term suppression.

To our knowledge, time-frequency analysis applied to scattered signals from concealed targets has also not been extensively treated in the literature. Gaunaurd and Nguyen [31] applied time-frequency analysis to the scattering from landmines buried under soil with known dielectric properties. Both simulation and measurement data were presented.

According to [21] and [27], different scattering mechanisms will appear differently in the time-frequency domain. The four main scattering phenomena are shown in Figures 1a to $1 \mathrm{~d}$. A vertical line in the time-frequency domain indicates that the scattering event occurs for a particular time instant but over all frequencies of excitation, and corresponds to wavefront phenomena, or a so-called scattering center (Figure 1a). A horizontal line in the time-frequency domain indicates that the scattering phenomenon dominates at a particular frequency over the transient time frame, and corresponds to a resonant mode (Figure 1b). However, dispersive phenomena appear as slopes in the time-frequency domain. A study of the transient scattering from a coated strip with a gap found that a positive slope in the time-frequency domain corresponds to a surfacewave mechanism due to the material coating (Figure 1c) [25]. Similarly, another study on the scattering of an opened

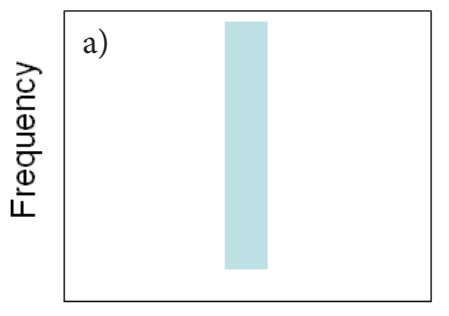

Time

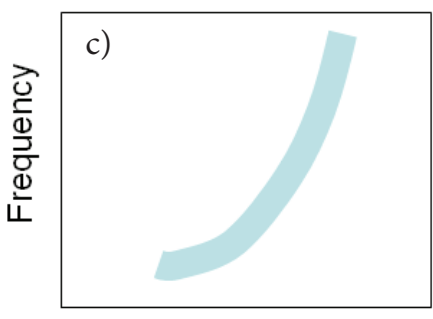

Time

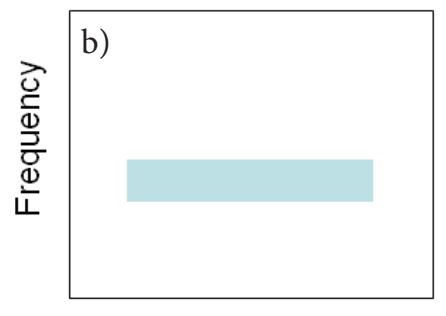

Time

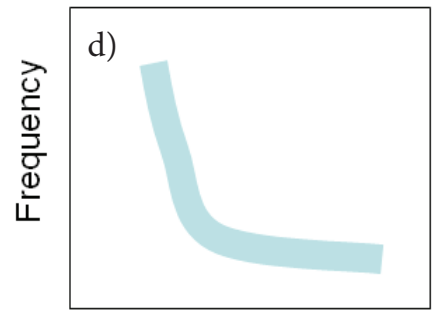

Time
Figure 1. Electromagnetic scattering mechanisms manifested in the time-frequency domain: (a) wavefront, (b) resonance, (c) material dispersion, and (d) structural dispersion.

waveguide [28] discovered that a negative slope in the timefrequency domain corresponds to structural dispersion (Figure 1d). Here, our focus is on wavefront-related phenomena, resonance phenomena, and their interactions, which are the key scattering mechanisms according to the Hybrid Wavefront Singularity Expansion Method.

\section{Numerical Examples}

This section will be divided into three parts. In the first part, examples of a PEC wire target sited beneath a lossless half-space with different orientations are given. The aim here was to study how the interactions between the target and the medium interface vary as the target's orientation changes. One may argue that it is also important to study the time-frequency analysis of transient signatures of wire targets at different orientations in free space. Such a study has been previously conducted [43], and thus is not repeated here. Then, examples using the same wire target sited below a frequency-dependent lossy half-space, corresponding to a model of realistic human tissue, will be given. The aim in this second part was to study the variations of the scattering behavior as the dielectric properties changed. Lastly, time-frequency analysis of the scattering from a hip-prosthesis model sited at various depths below realistic a human-tissue model $[44,45]$ will be studied. Monitoring targetdepth changes of the prosthesis model below a half-space of human tissue using the E-pulse technique was discussed in [11-13]. Here, we would like to see how the target's signature actually changes as the target's depth varies. 


\subsection{Examples for a Wire Target Below a Lossless Half-Space}

A PEC wire target of length $L=14 \mathrm{~cm}$, sited below a lossless half-space as shown in Figure 2, was considered. The center of the wire was at a depth of $h=L=14 \mathrm{~cm}$ below the air-dielectric interface, with an orientation angle of $\varphi$. This was very similar to the study of Vitebskiy and Carin in [33]. Medium 1 was free space, while the dielectric properties of medium 2 were $\varepsilon_{r 2}=9$ and $\sigma_{2}=0 \mathrm{~S} / \mathrm{m}$. These figures incidentally were a simple approximation to the dielectric properties of human tissue under microwave-frequency excitation [45]. Wire targets with an orientation of $\varphi=0^{\circ}$ (vertical), $\varphi=30^{\circ}, \varphi=60^{\circ}$, and $\varphi=90^{\circ}$ (horizontal) were studied. Excitation was a parallel-polarized plane wave at a Brewster angle of $\theta_{B}=71.5^{\circ}$, allowing total transmission from free space into the half-space. The angle of refraction was calculated to be [46]

$$
\theta_{T}=\tan ^{-1}\left(\sqrt{\frac{9 \varepsilon_{0}}{\varepsilon_{0}}}\right)=18.4^{\circ},
$$

and the scattered field was measured in the back-scattered direction.

The scattering-event sequence of the scattering problem in Figure 2 is shown in Figure 3, and was the same as Figure 2 of [33]. To study the occurrences of each scattering event, the timing of each individual interaction is illustrated in Figures 4a and $4 \mathrm{~b}$, respectively. In Figure $4 \mathrm{a}, t_{0}$ corresponds to the time required for the electromagnetic pulse to travel from the interface to the leading end of the wire in the direction of $\theta_{T}$, and $t_{1}$ corresponds to the time required for the electromagnetic pulse to travel to the far end of the wire in the direction of $\theta_{T}$. In Figure $4 \mathrm{~b}, t_{2}$ corresponds to the time required for the induced current to propagate from one end of the wire to the other; $t_{a}$ and $t_{b}$ correspond to the time for the electromagnetic pulse to vertically propagate from the wire's ends to the interface. The timing of each individual interaction was calculated, and is tabulated in Table 1 for different orientations, $\varphi$. The timing information given in Table 1 was then used for calculating the timing of the scattering events given in Figure 3.

The target response was computed in the frequency domain using the full-wave Moment Method solver FEKO [47], from $4.88 \mathrm{MHz}$ to $10 \mathrm{GHz}$ with 2048 equally spaced samples, and windowed with a Gaussian window. For verification purposes, the bicycle pulse used in [33] was also be used to ensure the correctness of the target signatures. Mathematically, the frequency response of the Gaussian window [48] and of the bicycle window are given by [10]

$$
W_{g}(f)=\frac{1}{c} e^{-\left(\frac{\pi T f}{4 c}\right)^{2}} e^{-j 2 \pi f t_{0}},
$$

$$
W_{b}(f)=\left(\omega t_{i}\right)^{4} e^{|\omega| t_{i}}, \omega=2 \pi f
$$

where $t_{0}=10 \mathrm{~ns}$ and $1 / t_{i}=3.33 \mathrm{GHz}$. The corresponding timedomain expression in closed form for Equation (2) can be found in [48] in the time domain, but a similar expression for Equation (3) is not available. The frequency-domain and timedomain responses of the Gaussian window and the bicycle pulse are shown in Figure 5. Note that in the time domain, the Gaussian pulse starts at $t_{\text {ref }}=10 \mathrm{~ns}$, while the bicycle pulse starts at $t_{r e f}=0 \mathrm{~ns}$. Together with the timing of each individual scattering event given in Table 1, the occurrences of the scattering events at different orientations, $\varphi$, are given in Table 2.

The time-domain target signatures for the $14 \mathrm{~cm}$ wire sited beneath the lossless half-space with various orientations, with $\varphi=0^{\circ}, \varphi=30^{\circ}, \varphi=60^{\circ}$, and $\varphi=90^{\circ}$, are shown in Figure 6 to Figure 9 , respectively. The signatures windowed by the Gaussian window are shown in Figures 6a, 7a, 8a, and 9a, while the signatures windowed by the bicycle window are shown in Figures $6 \mathrm{~b}, 7 \mathrm{~b}, 8 \mathrm{~b}$, and $9 \mathrm{~b}$, respectively. The results shown in Figures $6 b, 7 b, 8 b$, and $9 b$ were very similar to those of [33]. Furthermore, occurrences of various scattering events were clearly identified in both the Gaussian-windowed and bicyclewindowed target signatures, verifying the correctness of the target signatures, at least for similar data. For $\varphi=0^{\circ}$ and $\varphi=30^{\circ}$, events A, B, F, G, and $\mathrm{H}$ were not identified, due to their relatively small amplitudes.

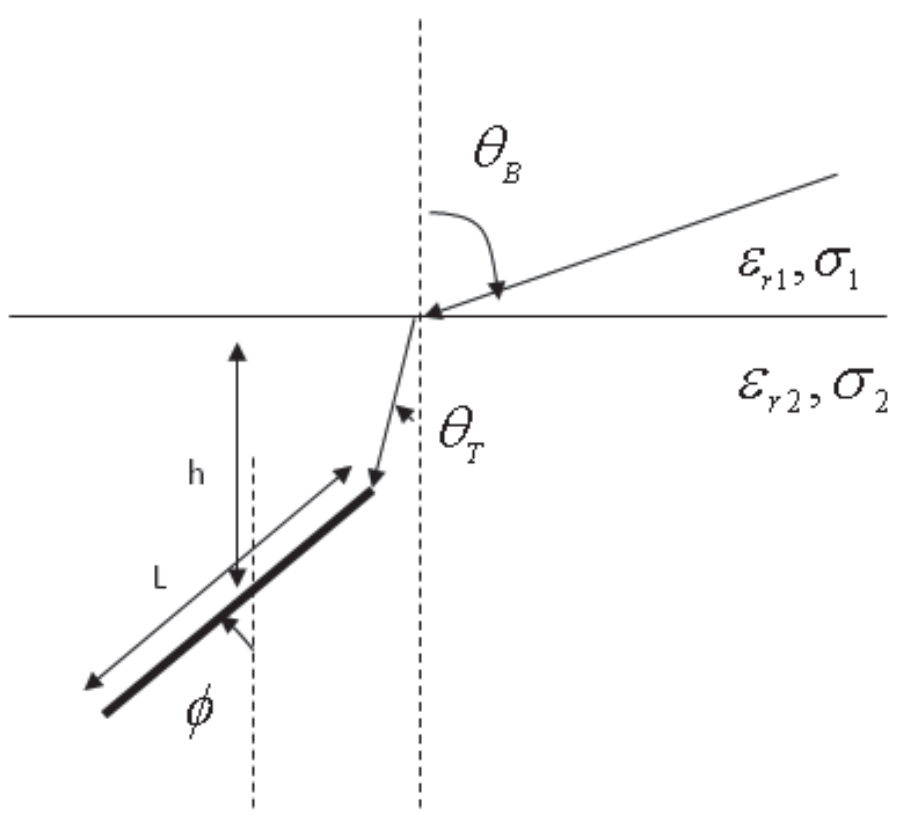

Figure 2. A PEC wire target sited beneath a half-space with the Brewster angle as the angle of incidence. The length of the wire target was $L$, and the orientation angle of the wire was $\varphi$. The depth of the wire, $h$, was the vertical distance between the center of the wire and the interface. 

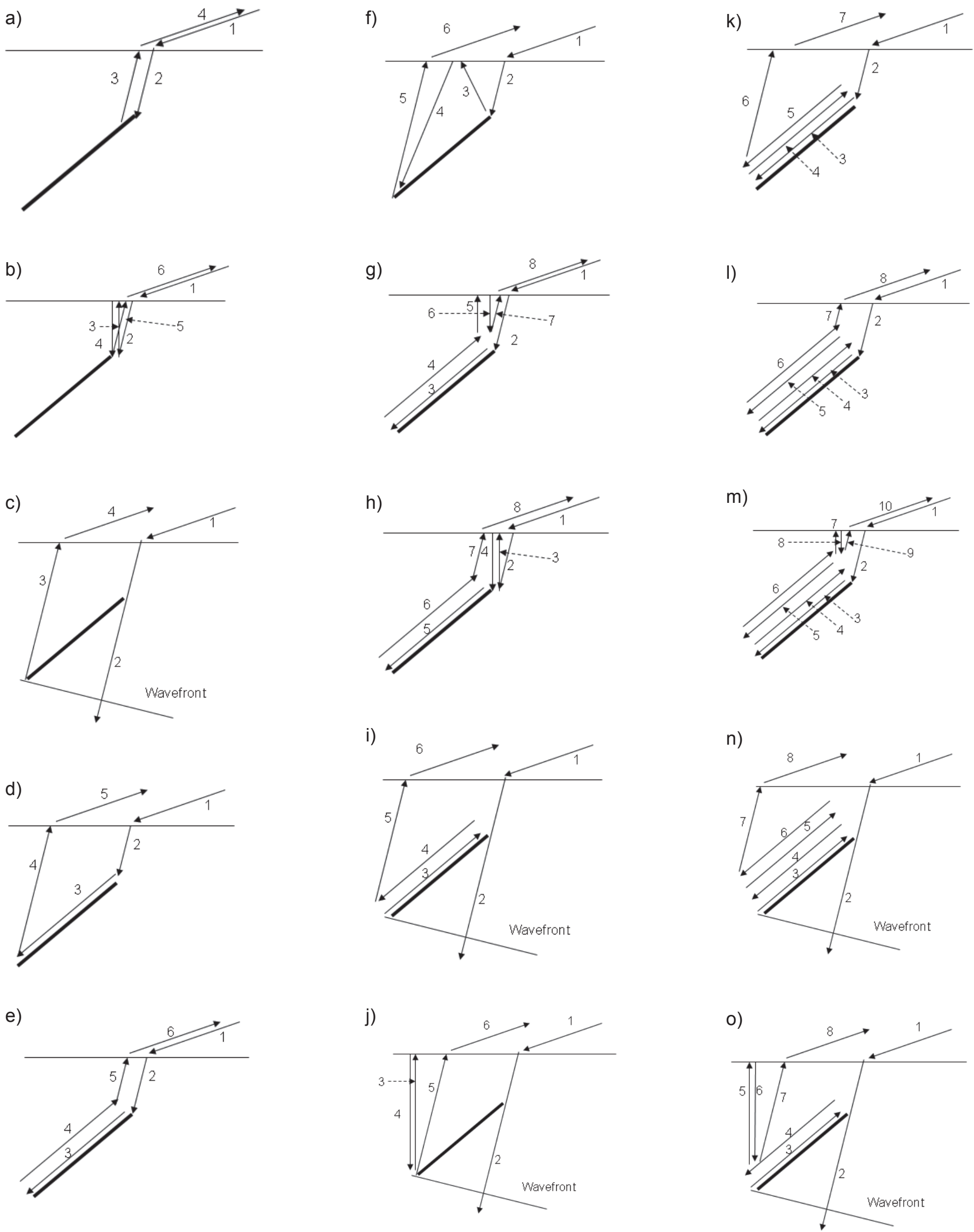

Figure 3. The scattering-event sequence of the wire target sited below the half-space with an incident plane wave at the Brewster angle of incidence. We considered 10 events, as shown in Figures 3a to 3o (identified as events A, B, ..., O, respectively), and the corresponding times of occurrence are shown in Table 2. The numbers in each figure indicate the order of how the electromagnetic wave traveled. 
Table 1. The time required for the occurrence of the individual wire-interface interaction events shown in Figure 4.

\begin{tabular}{|l|c|c|c|c|}
\hline Orientation & $\varphi=0^{\circ}$ & $\varphi=30^{\circ}$ & $\varphi=60^{\circ}$ & $\varphi=90^{\circ}$ \\
\hline \hline (Gaussian) & $10 \mathrm{~ns}$ & $10 \mathrm{~ns}$ & $10 \mathrm{~ns}$ & $10 \mathrm{~ns}$ \\
\hline (Bicycle) & $0 \mathrm{~ns}$ & $0 \mathrm{~ns}$ & $0 \mathrm{~ns}$ & $0 \mathrm{~ns}$ \\
\hline$t_{0}$ & $0.74 \mathrm{~ns}$ & $1.11 \mathrm{~ns}$ & $0.95 \mathrm{~ns}$ & $1.48 \mathrm{~ns}$ \\
\hline$t_{1}$ & $2.07 \mathrm{~ns}$ & $2.15 \mathrm{~ns}$ & $2.21 \mathrm{~ns}$ & $1.92 \mathrm{~ns}$ \\
\hline$t_{2}$ & $1.40 \mathrm{~ns}$ & $1.40 \mathrm{~ns}$ & $1.40 \mathrm{~ns}$ & $1.40 \mathrm{~ns}$ \\
\hline$t_{a}$ & $0.70 \mathrm{~ns}$ & $1.05 \mathrm{~ns}$ & $0.91 \mathrm{~ns}$ & $1.40 \mathrm{~ns}$ \\
\hline$t_{b}$ & $2.10 \mathrm{~ns}$ & $1.75 \mathrm{~ns}$ & $1.90 \mathrm{~ns}$ & $1.40 \mathrm{~ns}$ \\
\hline
\end{tabular}

Table 2. Occurrences of the various scattering events shown in Figure 3. The Gaussian pulse started at $t_{\text {ref }}=10$ ns and the bicycle pulse started at $t_{r e f}=0 \mathrm{~ns}$. Depending on the choice of the pulse in the time domain, the occurrence of the scattering events $A$ to $O$ was the sum of $t_{r e f}$ and the corresponding values shown in the table.

\begin{tabular}{|c|c|c|c|c|}
\hline Event & $\varphi=0^{\circ}$ & $\varphi=30^{\circ}$ & $\varphi=60^{\circ}$ & $\varphi=90^{\circ}$ \\
\hline \hline $\mathrm{A}$ & $1.48 \mathrm{~ns}$ & $1.67 \mathrm{~ns}$ & $2.21 \mathrm{~ns}$ & $2.95 \mathrm{~ns}$ \\
\hline $\mathrm{B}$ & $2.88 \mathrm{~ns}$ & $3.26 \mathrm{~ns}$ & $4.31 \mathrm{~ns}$ & $5.75 \mathrm{~ns}$ \\
\hline $\mathrm{C}$ & $4.13 \mathrm{~ns}$ & $4.42 \mathrm{~ns}$ & $4.31 \mathrm{~ns}$ & $3.84 \mathrm{~ns}$ \\
\hline $\mathrm{D}$ & $4.20 \mathrm{~ns}$ & $4.45 \mathrm{~ns}$ & $4.66 \mathrm{~ns}$ & $4.79 \mathrm{~ns}$ \\
\hline $\mathrm{E}$ & $4.28 \mathrm{~ns}$ & $4.47 \mathrm{~ns}$ & $5.01 \mathrm{~ns}$ & $5.75 \mathrm{~ns}$ \\
\hline $\mathrm{F}$ & $5.61 \mathrm{~ns}$ & $6.09 \mathrm{~ns}$ & $6.52 \mathrm{~ns}$ & $6.79 \mathrm{~ns}$ \\
\hline $\mathrm{G}$ & $5.68 \mathrm{~ns}$ & $6.06 \mathrm{~ns}$ & $7.11 \mathrm{~ns}$ & $8.55 \mathrm{~ns}$ \\
\hline $\mathrm{H}$ & $5.60 \mathrm{~ns}$ & $6.03 \mathrm{~ns}$ & $6.76 \mathrm{~ns}$ & $7.59 \mathrm{~ns}$ \\
\hline $\mathrm{I}$ & $6.93 \mathrm{~ns}$ & $7.22 \mathrm{~ns}$ & $7.11 \mathrm{~ns}$ & $6.64 \mathrm{~ns}$ \\
\hline $\mathrm{J}$ & $8.33 \mathrm{~ns}$ & $8.43 \mathrm{~ns}$ & $7.81 \mathrm{~ns}$ & $6.64 \mathrm{~ns}$ \\
\hline $\mathrm{K}$ & $7.00 \mathrm{~ns}$ & $7.25 \mathrm{~ns}$ & $7.46 \mathrm{~ns}$ & $7.59 \mathrm{~ns}$ \\
\hline $\mathrm{L}$ & $7.08 \mathrm{~ns}$ & $7.27 \mathrm{~ns}$ & $7.81 \mathrm{~ns}$ & $8.55 \mathrm{~ns}$ \\
\hline $\mathrm{M}$ & $8.48 \mathrm{~ns}$ & $8.86 \mathrm{~ns}$ & $9.91 \mathrm{~ns}$ & $11.35 \mathrm{~ns}$ \\
\hline $\mathrm{N}$ & $9.73 \mathrm{~ns}$ & $10.02 \mathrm{~ns}$ & $9.91 \mathrm{~ns}$ & $9.44 \mathrm{~ns}$ \\
\hline $\mathrm{O}$ & $11.13 \mathrm{~ns}$ & $11.23 \mathrm{~ns}$ & $10.61 \mathrm{~ns}$ & $9.44 \mathrm{~ns}$ \\
\hline
\end{tabular}
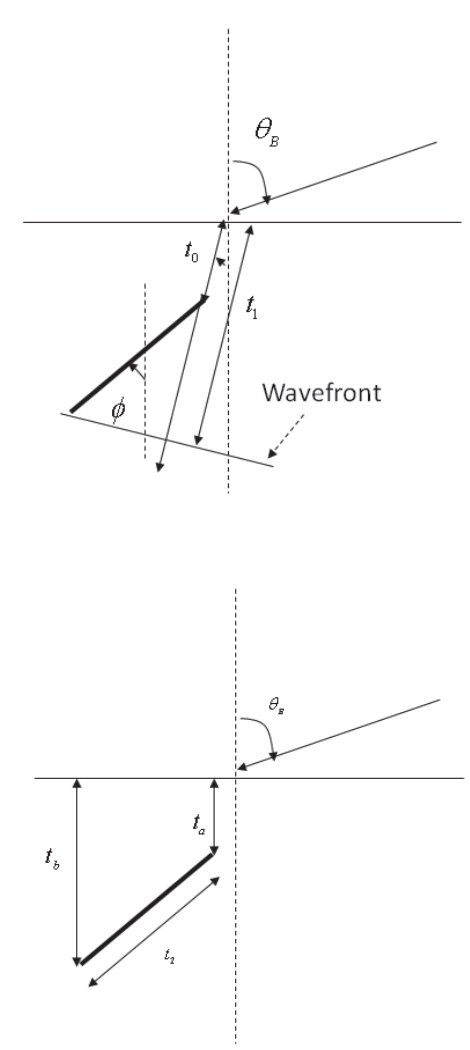

Figure 4. The various interactions of the electromagnetic wave between the interface and the wire target $(a$, top; $b$, bottom). 
(a)

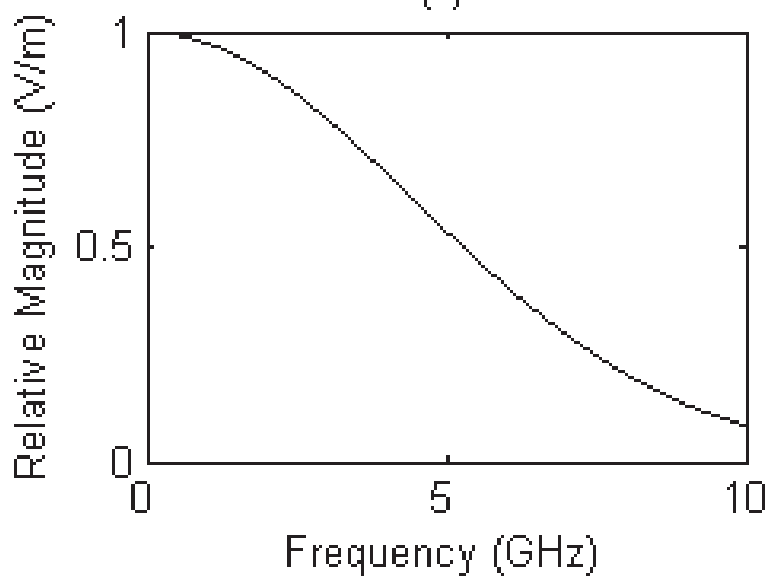

(c)

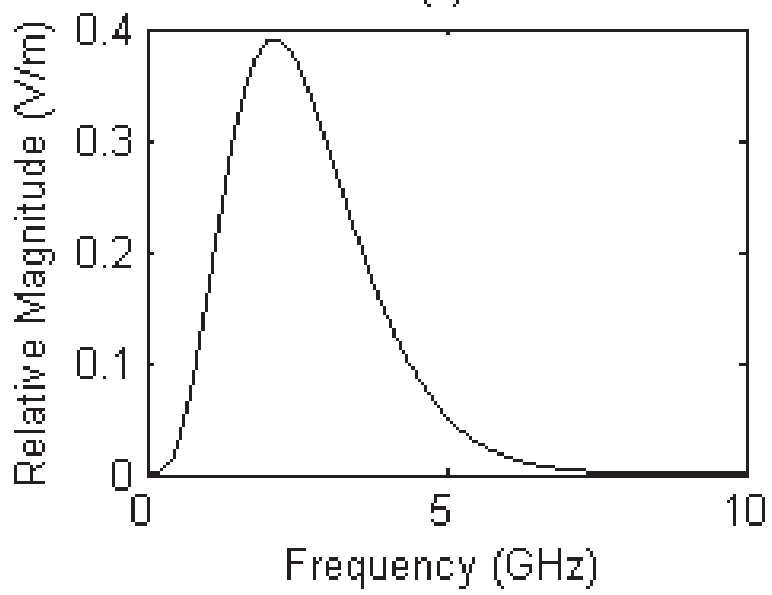

(b)

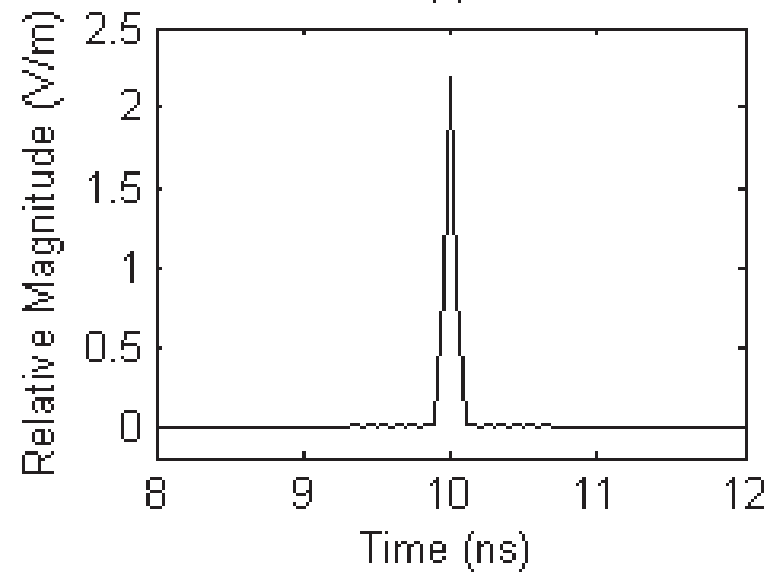

(d)

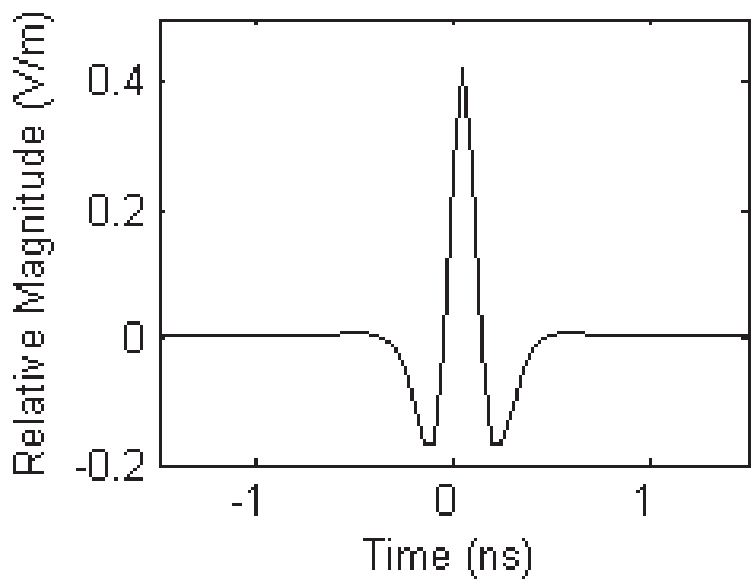

Figure 5. The time and frequency responses of the Gaussian- and bicycle-pulse windows: (a, top) the frequency- and (b) time-domain responses of the Gaussian windowing; (c) the frequency- and (d, bottom) time-domain responses of the bicyclepulse windowing.
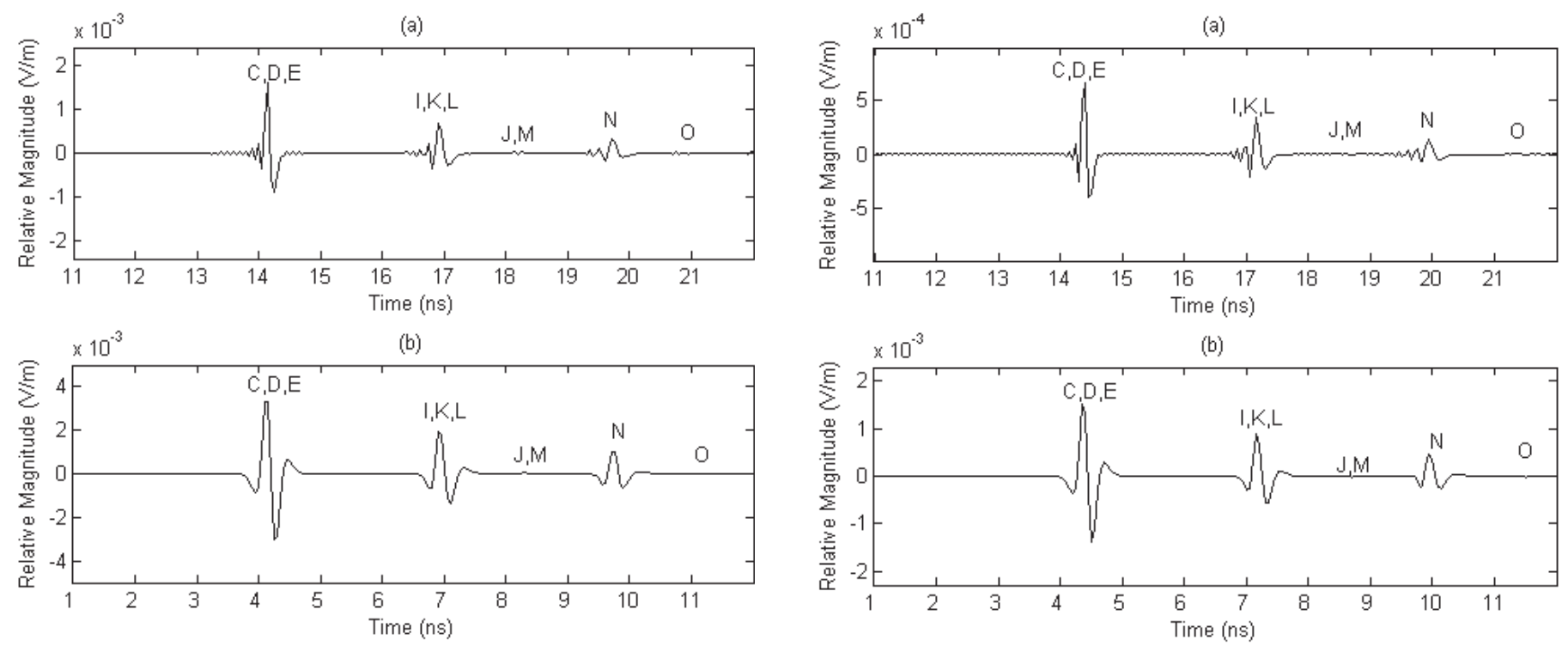

Figure 6. The time-domain signatures for the $14 \mathrm{~cm}$ wire target sited beneath a lossless half-space of $\varepsilon_{r}=9, \sigma=0$

$\mathrm{S} / \mathrm{m}$, at a depth of $14 \mathrm{~cm}$, with an orientation of $\varphi=0^{\circ}$. The time-domain signature was windowed using (a, top) a Gaussian window and (b) a bicycle window.

Figure 7. The time-domain signatures for the $14 \mathrm{~cm}$ wire target sited beneath a lossless half-space of $\varepsilon_{r}=9, \sigma=0$ $\mathrm{S} / \mathrm{m}$, at a depth of $14 \mathrm{~cm}$, with an orientation of $\varphi=30^{\circ}$. The time-domain signature was windowed using (a, top) a Gaussian window and (b) a bicycle window. 

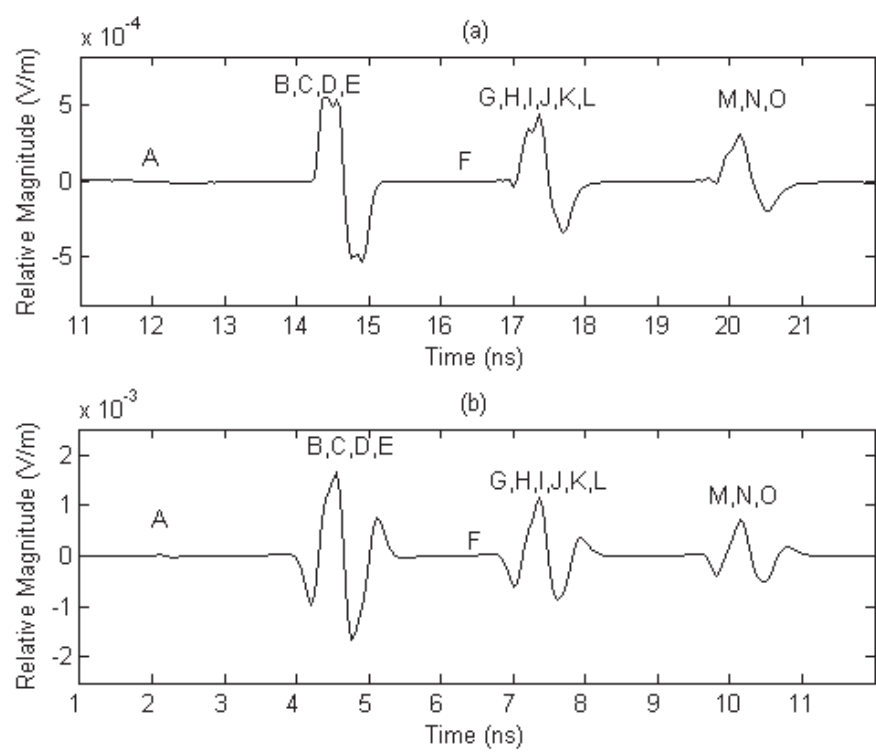

Figure 8. The time-domain signatures for the $14 \mathrm{~cm}$ wire target sited beneath a lossless half-space of $\varepsilon_{r}=9, \sigma=0$ $\mathrm{S} / \mathrm{m}$, at a depth of $14 \mathrm{~cm}$, with an orientation of $\varphi=60^{\circ}$. The time-domain signature was windowed using (a, top) a Gaussian window and (b) a bicycle window.
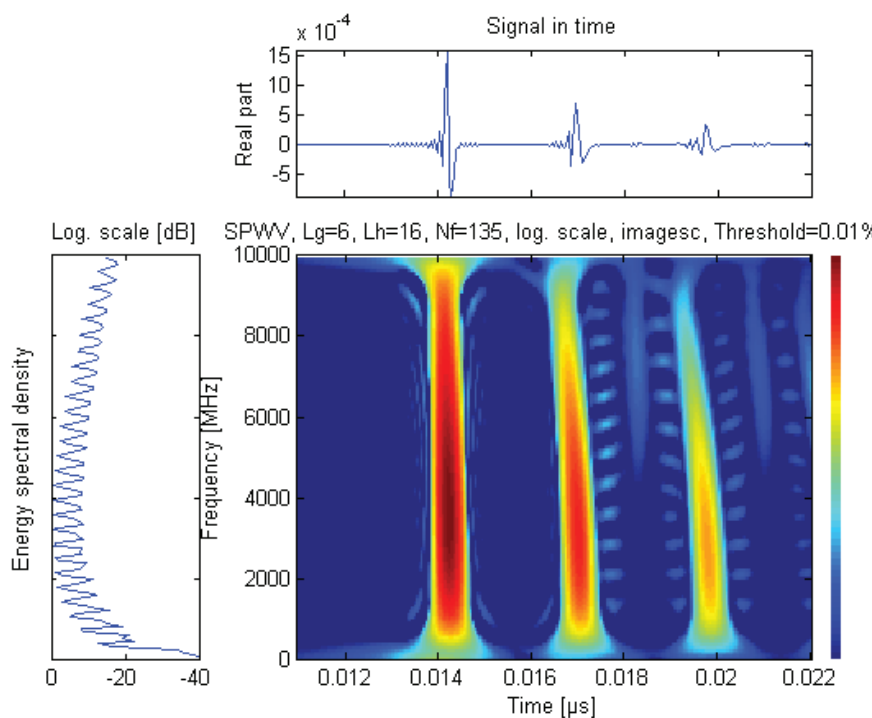

Figure 10a. The smooth pseudo-Wigner-Ville distribution of the target's signature for the $14 \mathrm{~cm}$ wire target sited beneath a lossless half-space of $\varepsilon_{r}=9, \sigma=0 \mathrm{~S} / \mathrm{m}$ at a depth of $14 \mathrm{~cm}$ with an orientation of $\varphi=0^{\circ}$. The-time domain signature was windowed using a Gaussian window.
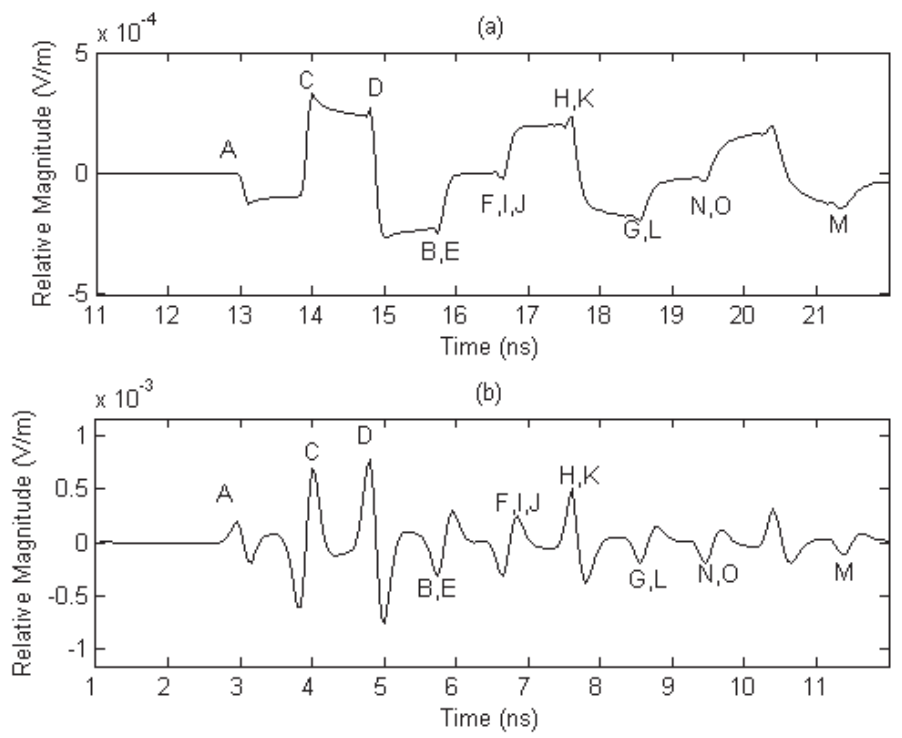

Figure 9. The time-domain signatures for the $14 \mathrm{~cm}$ wire target sited beneath a lossless half-space of $\varepsilon_{r}=9, \sigma=0$ $\mathrm{S} / \mathrm{m}$, at a depth of $14 \mathrm{~cm}$, with an orientation of $\varphi=90^{\circ}$. The time-domain signature was windowed using (a, top) a Gaussian window and (b) a bicycle window.
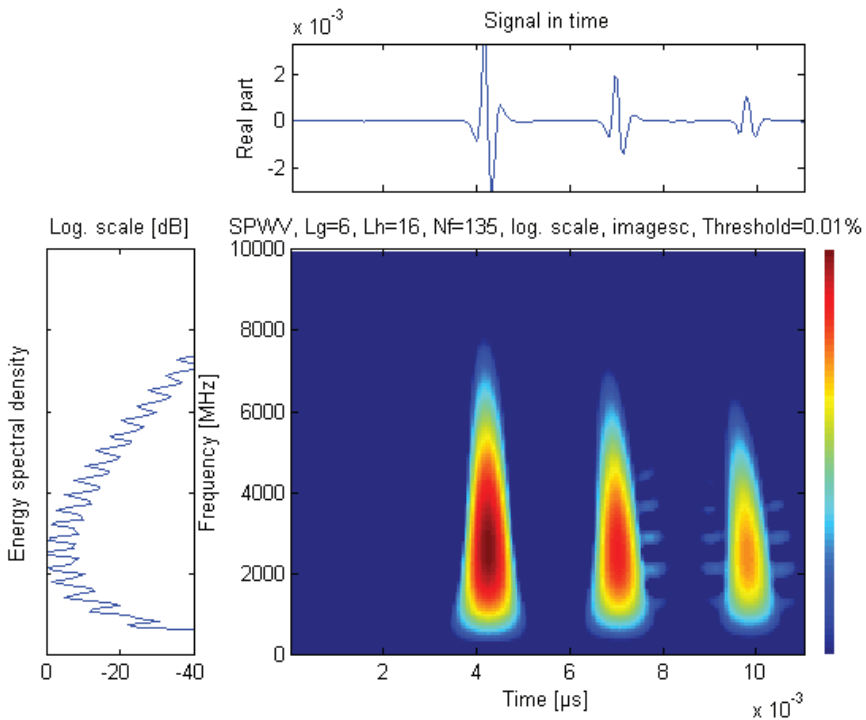

Figure 10b. The smooth pseudo-Wigner-Ville distribution of the target's signature for the $14 \mathrm{~cm}$ wire target sited beneath a lossless half-space of $\varepsilon_{r}=9, \sigma=0 \mathrm{~S} / \mathrm{m}$ at a depth of $14 \mathrm{~cm}$ with an orientation of $\varphi=0^{\circ}$. The-time domain signature was windowed using a bicycle window. 
Next, the time-frequency distributions (TFDs) of the target signatures, with both Gaussian and bicycle windowing, are shown in Figures 10 to 13. Based on the observations in our previous study [30], the smooth pseudo-Wigner-Ville distribution (SPWVD) was found to be preferred over the others (in terms of resolution and reasonable capabilities of suppression of cross-terms), and they were thus chosen for this example. In this paper, all the time-frequency distributions were computed using the toolbox developed by CNRS in France and Rice University in the USA [41]. For $\varphi=0^{\circ}, \varphi=30^{\circ}$, and $\varphi=60^{\circ}$

, there were three inclined vertical lines shown in the timefrequency domain within the timeframe. These inclined vertical lines corresponded to the occurrences of the wavefrontscattering events, and there were no horizontal lines joining them, indicating that they did not have strong interactions with each other. In this example, one would expect a number of horizontal lines that corresponded to the resonant modes of this strongly resonating target, with a large number of sharp peaks, as shown in the frequency responses on the left of each figure. In fact, this can be explained by the Hybrid Wavefront Singularity Expansion Method. Under the Hybrid Wavefront Singularity Expansion Method description of $[19,20]$, it was shown and proven mathematically that wavefronts and resonances form alternative descriptions for transient scattering by targets. Various diffraction events (wavefronts) occur in the late time period, can be thought of as synthesizing the scattered field, and can be summed collectively into Singularity Expansion Method resonances, and vice versa. Here, one can imagine that the large number of resonant modes for the wire target at the orientations of $\varphi=0^{\circ}$ and $\varphi=30^{\circ}$ collectively synthesized the late-time wavefront events. Comparing the results for the two different types of windowing, the bicycle windowing had a lower cutoff frequency and, consequently, high-frequency components were not observed in the frequency domain as well as in the time-frequency domain.

As shown in the smooth pseudo-Wigner-Ville distributions of Figures 13a and $13 \mathrm{~b}$ for $\varphi=90^{\circ}$, there were a number of vertical lines corresponding to the occurrence of the scattering events. They were joined by a number of horizontal lines below $5 \mathrm{GHz}$, indicating that the scattering events strongly interacted with each other. However, owing to the different shapes of the frequency responses of the Gaussian and bicycle windowing, the relative amplitudes of the resonant peaks were different, and thus different energy distributions were observed between the two cases in the time-frequency domain. Lastly, there were also a number of horizontal lines shown in the smooth pseudo-Wigner-Ville distributions above $5 \mathrm{GHz}$, in Figure 13. According to the conclusions from [30], these horizontal lines were cross-term interferences. This can be confirmed by the fact that they did not appear in the spectrograms of Figures 14a and 14b for the same target response using Gaussian and bicycle windowing.

To probe further, the transient electromagnetic scattering of the same wire target inside a homogenous environment of $\varepsilon_{r}=9$ and $\sigma=0 \mathrm{~S} / \mathrm{m}$ at different orientations was considered. We wanted to see how the response differed compared to the case of no air-medium discontinuity. In the computation, the incident aspects and polarizations of the incident and scattered fields, as well as the orientation of the wire, remained the same. The half-space Green's function was replaced by a homogenous Green's function. The target's responses were computed in the same manner, and the smooth pseudo-Wigner-Ville distributions are shown in Figure 15. When the orientation of the wire was $\varphi=0^{\circ}$ (Figure 15a), mainly only the resonant events were observed. When the orientation changed to $\varphi=30^{\circ}, \varphi=60^{\circ}$, and $\varphi=90^{\circ}$, both horizontal and vertical lines appeared in the time-frequency domain. Such an observation was in line with the findings in the early work on time-frequency analysis of targets in free space, and the interactions of the wavefront and resonant events under the Hybrid Wavefront Singularity Expansion Method description [16-20, 30]. By comparing with the case of the air-medium discontinuity, it was observed that there were more interactions between the wavefront and the resonant events when the wire was inside a homogenous environment. The results indicated that the introduction of the air-medium interface suppressed some of the resonant and wavefront events.

While not presented here, an attempt was also made to compute the same wire target buried at depths from $2 \mathrm{~cm}$ to $18 \mathrm{~cm}$ below the air-medium interface. As expected, the occurrences of the scattering events were delayed as the depth increased. Visually, there were no significant changes of the vertical positions of the horizontal lines that corresponded to the resonant events. This could be explained by the fact that the resonant frequencies varied slightly (within a few $\mathrm{MHz}$ ) as the target depth changed [7-10]. At the same time, the resonant modes mainly corresponded to the wire target's "self resonance." Resonant modes that corresponded to interactions between the target and the air-medium interface - "image resonance" - were not well observed in the time-frequency plots. This was probably because the residues of those resonant modes were relatively low compared to the self-resonance.

\subsection{Examples of a Wire Target Beneath a Frequency-Dependent Lossy Half-Space}

A time-frequency analysis of the same wire target sited below a lossy half-space with different dielectric properties was considered. In particular, realistic human-tissue models, using the Cole-Cole-4 and Debye models, were employed [44, 45]. The relative permittivity and conductivity, as functions of frequency up to $10 \mathrm{GHz}$, of the fat-infiltrated human-tissue model and the dry-skin model, are shown in Figures 16a and $16 \mathrm{~b}$, respectively. The attenuation constant of the human-tissue model can be given as [46]

$$
\alpha=\omega \sqrt{\mu \varepsilon_{0} \varepsilon_{r}}\left\{\frac{1}{2}\left[\sqrt{1+\left(\frac{\sigma}{\omega \varepsilon_{0} \varepsilon_{r}}\right)^{2}}-1\right]\right\}^{1 / 2},
$$

where $\omega=2 \pi f$, and $\sigma$ and $\varepsilon_{r}$ are the conductivity and relative permittivity of the dielectric, respectively. The attenuation 


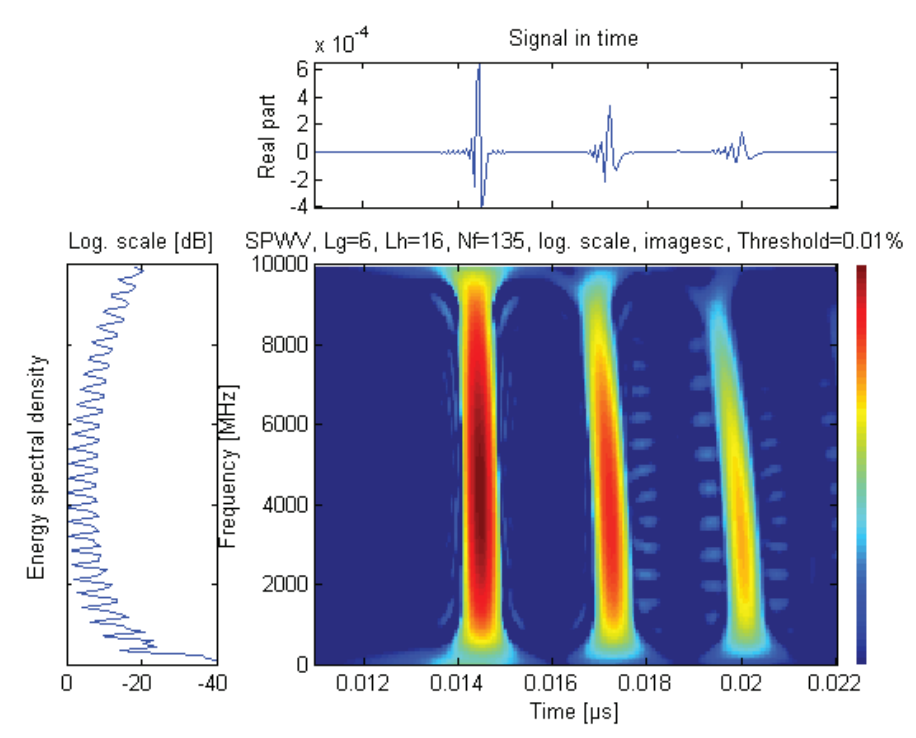

Figure 11a. The smooth pseudo-Wigner-Ville distribution of the target's signature for the $14 \mathrm{~cm}$ wire target sited beneath a lossless half-space of $\varepsilon_{r}=9, \sigma=0 \mathrm{~S} / \mathrm{m}$ at a depth of $14 \mathrm{~cm}$ with an orientation of $\varphi=30^{\circ}$. The-time domain signature was windowed using a Gaussian window.
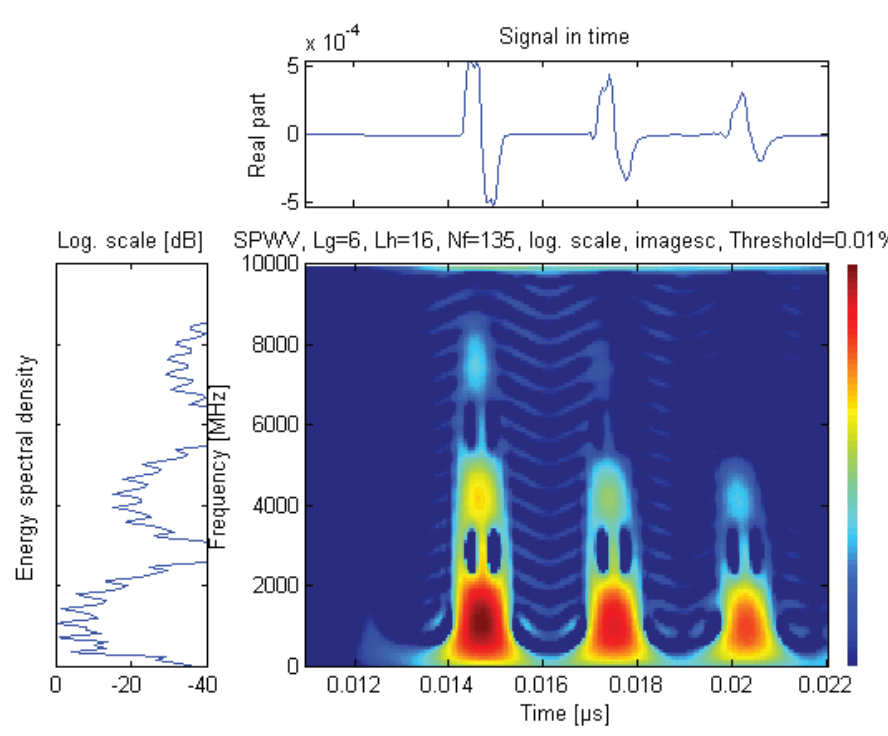

Figure 12a. The smooth pseudo-Wigner-Ville distribution of the target's signature for the $14 \mathrm{~cm}$ wire target sited beneath a lossless half-space of $\varepsilon_{r}=9, \sigma=0 \mathrm{~S} / \mathrm{m}$ at a depth of $14 \mathrm{~cm}$ with an orientation of $\varphi=60^{\circ}$. The-time domain signature was windowed using a Gaussian window.
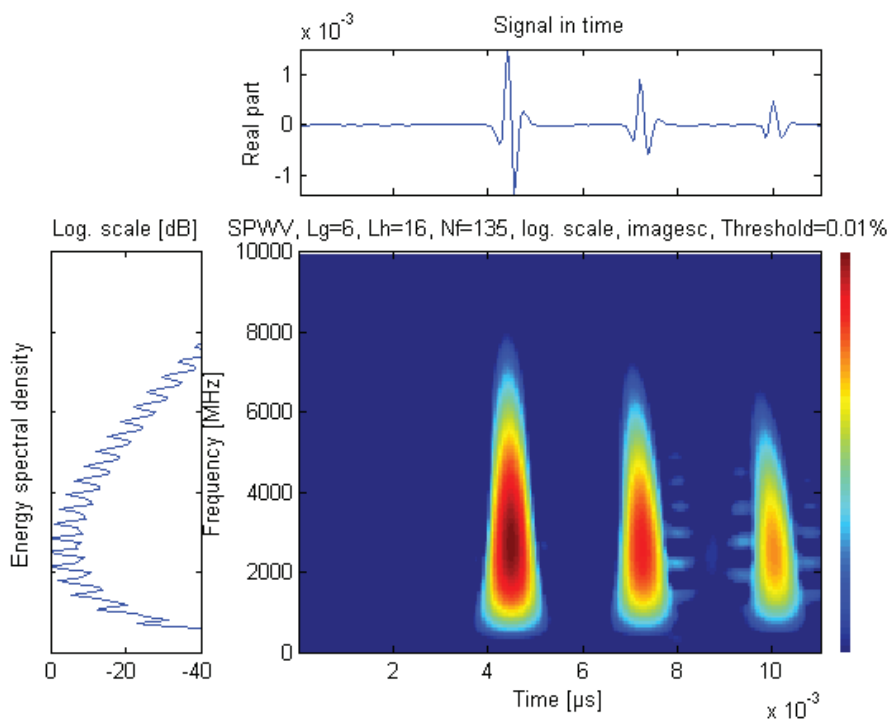

Figure 11b. The smooth pseudo-Wigner-Ville distribution of the target's signature for the $14 \mathrm{~cm}$ wire target sited beneath a lossless half-space of $\varepsilon_{r}=9, \sigma=0 \mathrm{~S} / \mathrm{m}$ at a depth of $14 \mathrm{~cm}$ with an orientation of $\varphi=30^{\circ}$. The-time domain signature was windowed using a bicycle window.
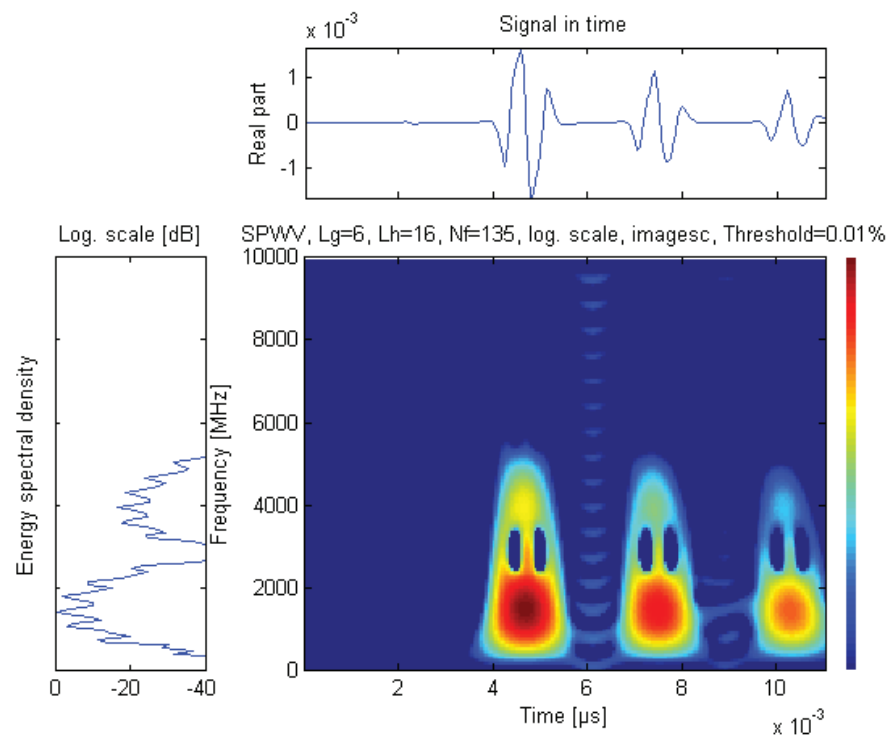

Figure 12b. The smooth pseudo-Wigner-Ville distribution of the target's signature for the $14 \mathrm{~cm}$ wire target sited beneath a lossless half-space of $\varepsilon_{r}=9, \sigma=0 \mathrm{~S} / \mathrm{m}$ at a depth of $14 \mathrm{~cm}$ with an orientation of $\varphi=60^{\circ}$. The-time domain signature was windowed using a bicycle window. 

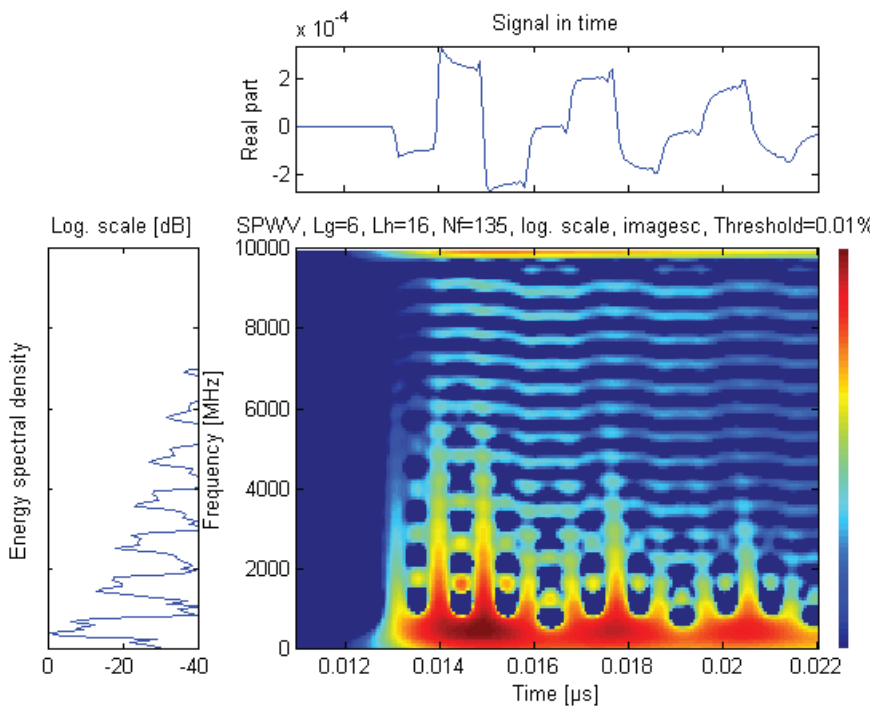

Figure 13a. The smooth pseudo-Wigner-Ville distribution of the target's signature for the $\mathbf{1 4} \mathbf{~ c m}$ wire target sited beneath a lossless half-space of $\varepsilon_{r}=9, \sigma=0 \mathrm{~S} / \mathrm{m}$ at a depth of $14 \mathrm{~cm}$ with an orientation of $\varphi=90^{\circ}$. The-time domain signature was windowed using a Gaussian window.
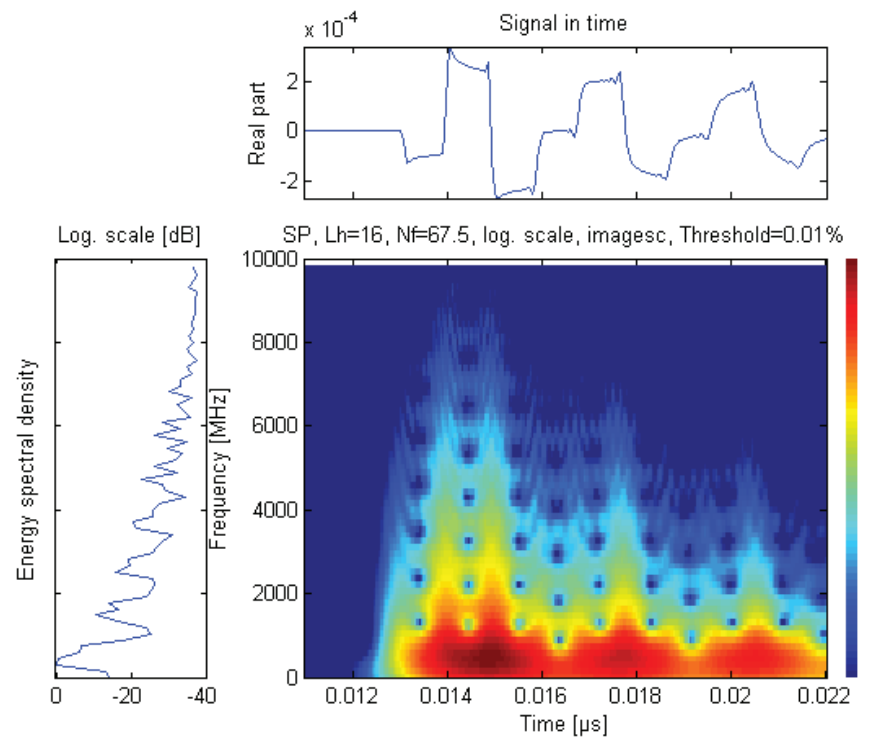

Figure 14a. The spectrogram of the target's signature for the $\mathbf{1 4} \mathbf{~ c m}$ wire target sited beneath a lossless half-space of $\varepsilon_{r}=9, \sigma=0 \mathrm{~S} / \mathrm{m}$ at a depth of $14 \mathrm{~cm}$ with an orientation of $\varphi=90^{\circ}$. The time-domain signature was windowed using a Gaussian window.
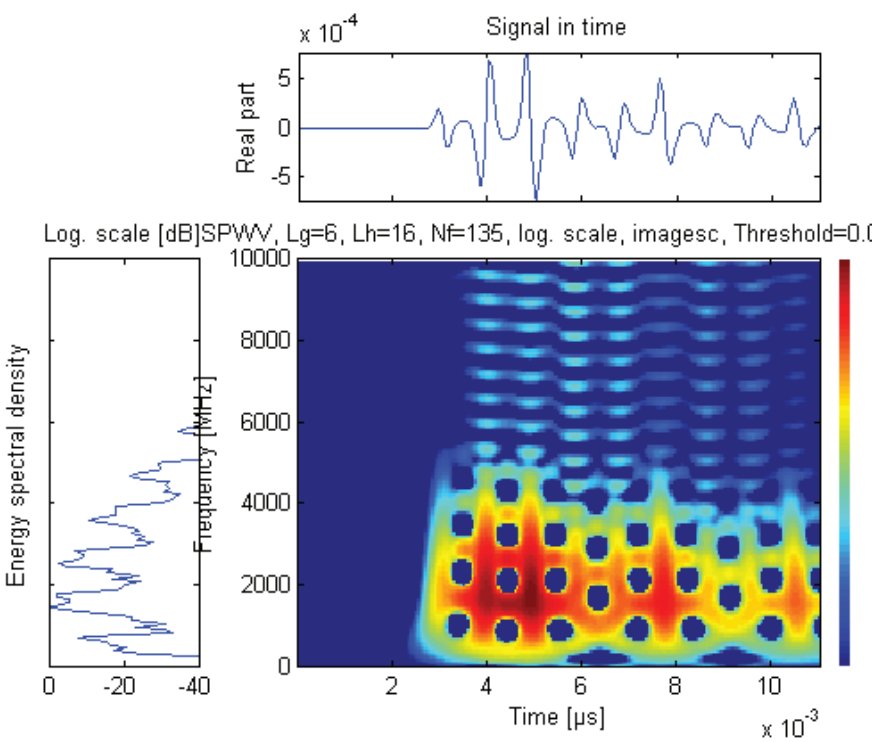

Figure 13b. The smooth pseudo-Wigner-Ville distribution of the target's signature for the $14 \mathrm{~cm}$ wire target sited beneath a lossless half-space of $\varepsilon_{r}=9, \sigma=0 \mathrm{~S} / \mathrm{m}$ at a depth of $14 \mathrm{~cm}$ with an orientation of $\varphi=90^{\circ}$. The-time domain signature was windowed using a bicycle window.
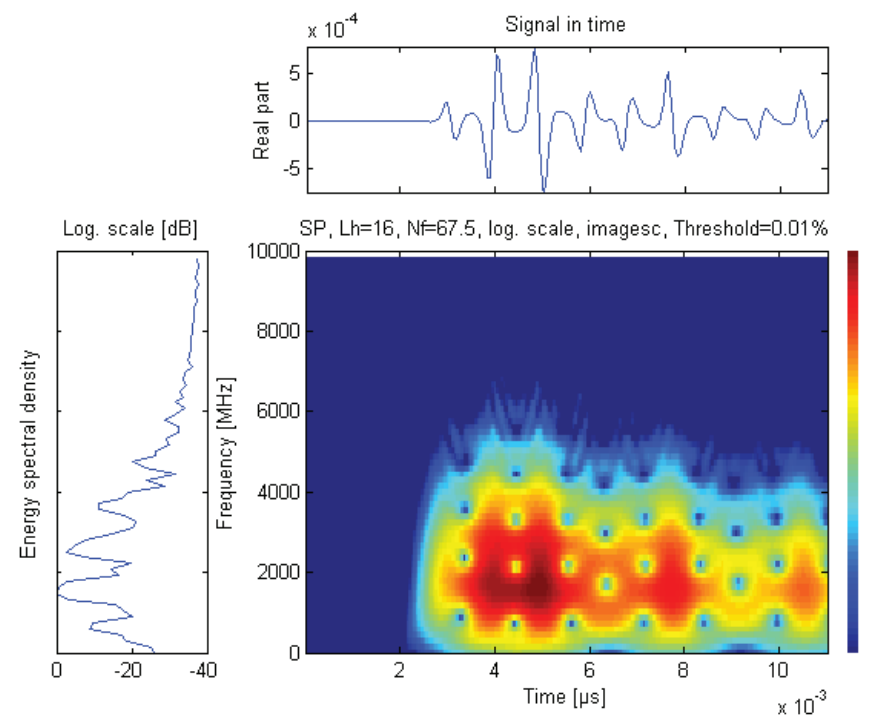

Figure 14b. The spectrogram of the target's signature for the $14 \mathrm{~cm}$ wire target sited beneath a lossless half-space of $\varepsilon_{r}=9, \sigma=0 \mathrm{~S} / \mathbf{m}$ at a depth of $14 \mathrm{~cm}$ with an orientation of $\varphi=90^{\circ}$. The time-domain signature was windowed using a bicycle window. 

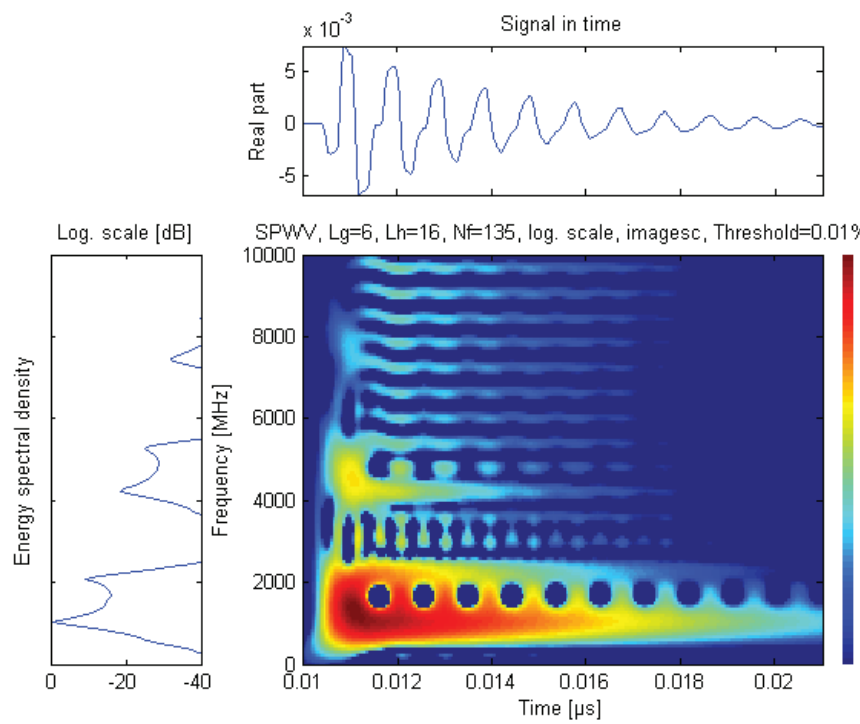

Figure 15a. The smooth pseudo-Wigner-Ville distribution of the target's signature for the $14 \mathrm{~cm}$ wire target inside a lossless environment of $\varepsilon_{r}=9, \sigma=0 \mathrm{~S} / \mathrm{m}$ with an orientation of $\varphi=0^{\circ}$. The time-domain signature was windowed using a Gaussian window.
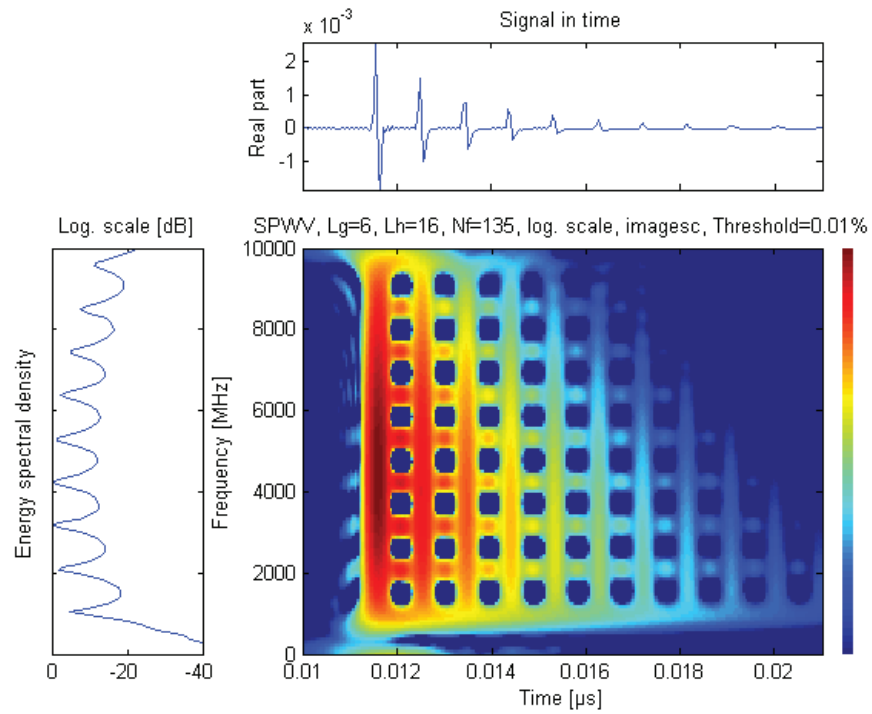

Figure 15c. The smooth pseudo-Wigner-Ville distribution of the target's signature for the $14 \mathrm{~cm}$ wire target inside a lossless environment of $\varepsilon_{r}=9, \sigma=0 \mathrm{~S} / \mathrm{m}$ with an orientation of $\varphi=60^{\circ}$. The time-domain signature was windowed using a Gaussian window.
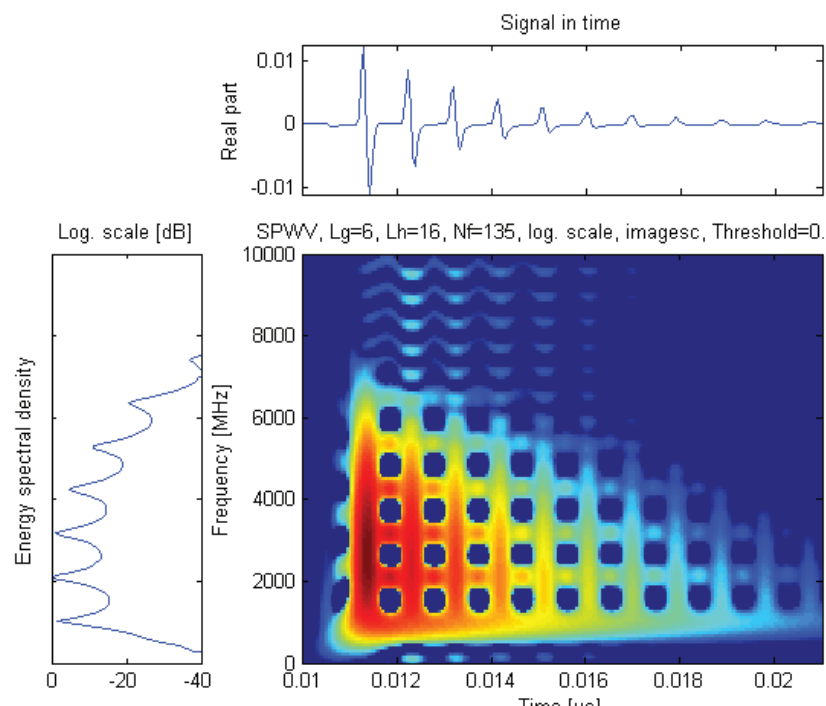

SPW, $L g=6, L h=16, N f=135, \log$. scale, imagesc, Threshold $=0.01 \%$

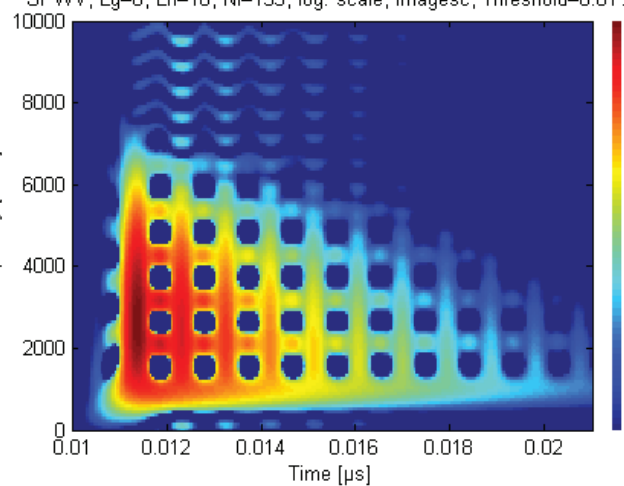

Figure 15b. The smooth pseudo-Wigner-Ville distribution of the target's signature for the $\mathbf{1 4} \mathbf{~ c m}$ wire target inside a lossless environment of $\varepsilon_{r}=9, \sigma=0 \mathrm{~S} / \mathrm{m}$ with an orientation of $\varphi=30^{\circ}$. The time-domain signature was windowed using a Gaussian window.
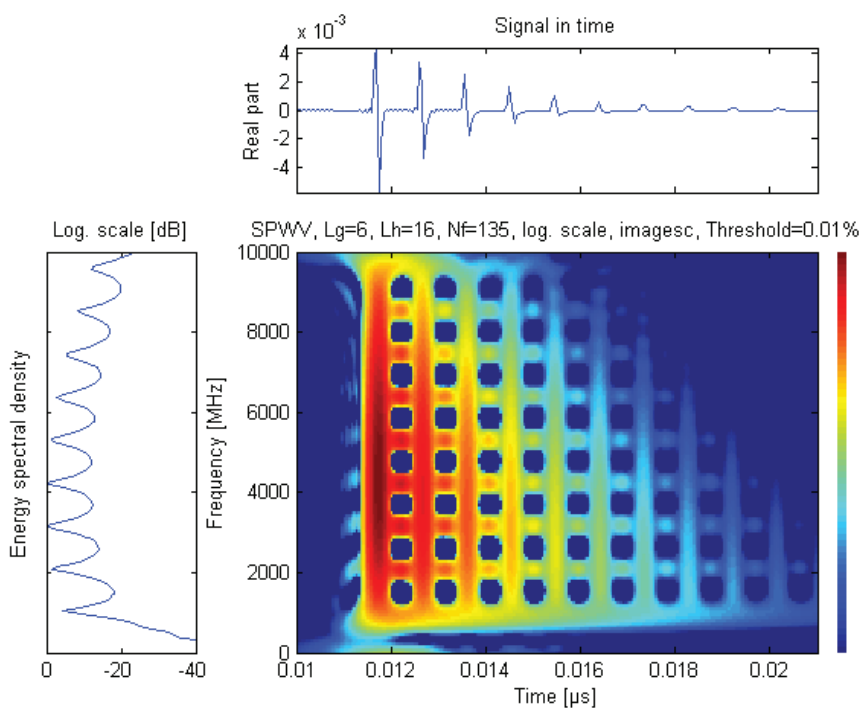

Figure 15d. The smooth pseudo-Wigner-Ville distribution of the target's signature for the $\mathbf{1 4} \mathbf{~ c m}$ wire target inside a lossless environment of $\varepsilon_{r}=9, \sigma=0 \mathrm{~S} / \mathrm{m}$ with an orientation of $\varphi=90^{\circ}$. The time-domain signature was windowed using a Gaussian window. 

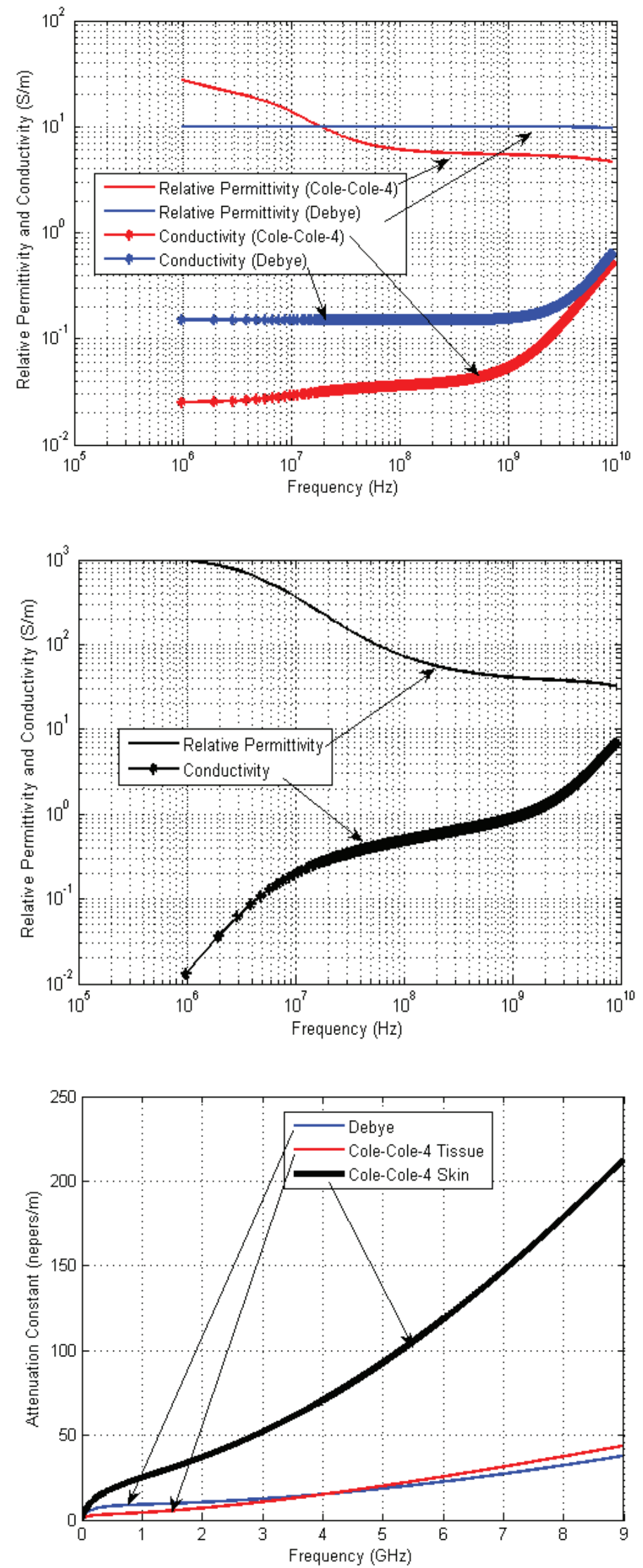

Figure 16. Dielectric profiles of the (a, top) Debye and Cole-Cole-4 (fat-infiltrated tissue) human-tissue model, (b, middle) the Cole-Cole-4 dry-skin model, and (c, bottom) the attenuation factors as functions of frequency. factor of the human-tissue model as a function of frequency is shown in Figure 16c.

The time-domain responses for the same $14 \mathrm{~cm}$ wire sited below different human-tissue models are shown in Figure 16. The wire was horizontally oriented $\left(\varphi=90^{\circ}\right)$ at a depth of $14 \mathrm{~cm}$ below the air-dielectric interface. We meant to investigate the interaction between the wavefront and the resonance events in the time-frequency domain. The wire was again excited with a parallel-polarized plane wave at $\theta_{i}=71.5^{\circ}$, and the scattered field was measured in the back-scattered direction. Here, we simply used the Brewster angle of the lossless case for the lossy situation, as the Brewster angle of a lossy dielectric is not real [33]. Figures 17a, 17b, and 17c show the target's response for the wire below the fat-infiltrated human-tissue model, the fatinfiltrated tissue model with a $5 \mathrm{~mm}$ skin layer, and the Debye human-tissue model, respectively [44, 45].

As shown in Figure 17, the first few scattering events on the scattering path were clearly observed in the time domain. Here the target, target's depth, and target's orientation remained the same, and we expected similar results when comparing Figure 17 with Figure 9. However, owing to the frequencydependent nature of the dielectric properties of the human tissue, the angle of refraction, $\theta_{T}$, and the phase velocity of the transmitted wave varied as a function of frequency [32]. As a result, for the same frequency component, the wave reached the target at a slightly different time, and the ultra-wideband target response "smeared" in the time domain. On the other hand, the relative permittivity of the half-space was different from that in Section 4.1 such that the resonant frequencies were slightly different. In addition, the electromagnetic wave attenuated as it propagated within the lossy dielectric, such that the magnitude of the signal shown in Figure 17 was lower than that of Figure 9 for the lossless case. The higher-order scattering events were not clearly identified, as the magnitudes of the electromagnetic waves attenuated significantly after a few interactions between the target and the interface. Comparing the three cases shown in Figure 17, the wire below the fat-infiltrated model had the highest signal level. With the introduction of the $5 \mathrm{~mm}$ skin layer, the amplitude of the signal decreased, as the skin layer had a higher attenuation throughout the frequency range of interest, as shown in Figure 16c. The signal level for the wire below the Debye human-tissue model was the lowest. This was probably because the attenuation factor of the Debye model was higher than that of the fat-infiltrated human-tissue below $4 \mathrm{GHz}$ (Figure 16c).

Next, the time-frequency analysis of the target's signature is shown in Figures 18a to 18c. Compared to the lossless cases shown in Figure 13 and Figure 14, where the signal was made up of a dominant resonance at $\sim 700 \mathrm{MHz}$ with a number of partial resonances, the results here indicated that only the first dominant resonance was observed for the wire. There also appeared to be a "partial resonance" that occurred at $\sim 1.5 \mathrm{GHz}$ as observed for the Debye case of Figure 18a between $12 \mathrm{~ns}$ to $14 \mathrm{~ns}$; however, it was not as obvious as in the lossless case of Figure 13a. Again, the nature of these resonances is left for 


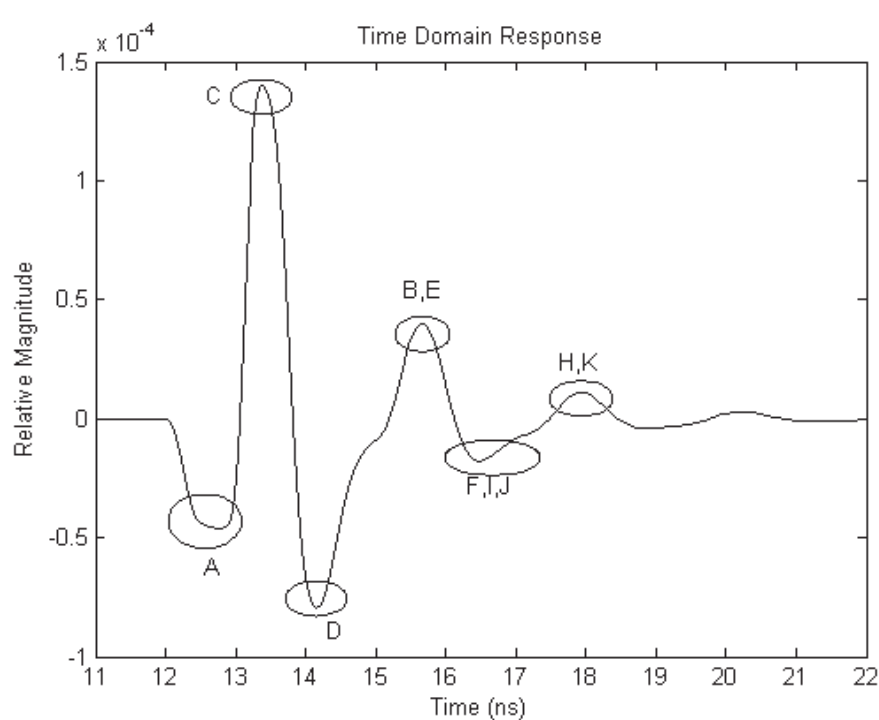

Figure 17a. The time-domain signatures for the $14 \mathrm{~cm}$ wire target sited beneath a half-space of fat-infiltrated tissue at a depth of $14 \mathrm{~cm}$ with an orientation of $\varphi=90^{\circ}$ (horizontal). The time-domain signature was windowed using a Gaussian window.

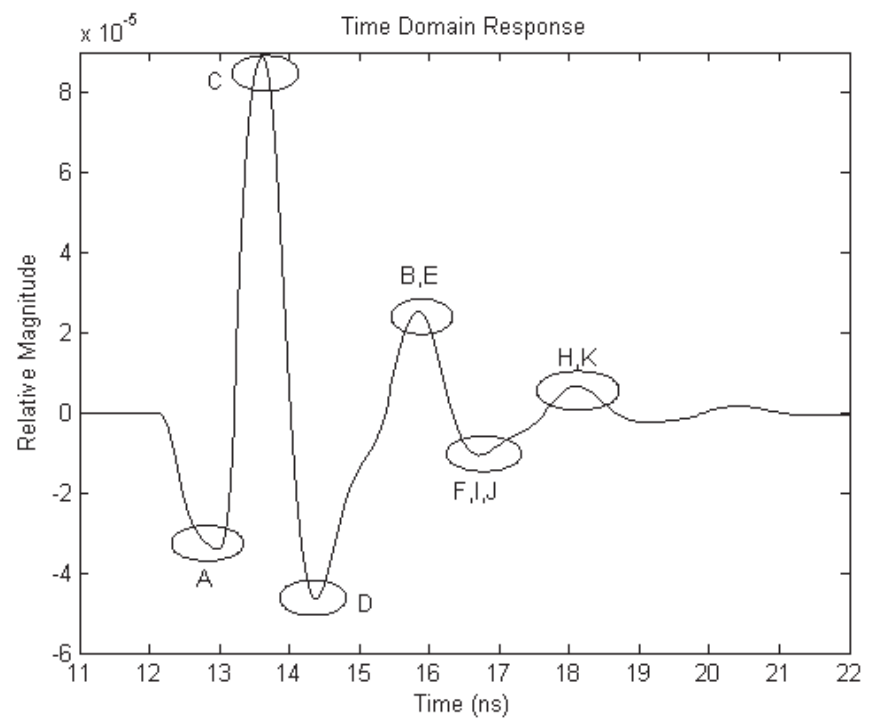

Figure 17b. The time-domain signature for the $14 \mathrm{~cm}$ wire target sited beneath a layered half-space of fat-infiltrated tissue with a $5 \mathrm{~mm}$ skin layer on top. The wire was sited at a depth of $14 \mathrm{~cm}$ below the air-skin interface with an orientation of $\varphi=90^{\circ}$ (horizontal). The time-domain signature was windowed using a Gaussian window.

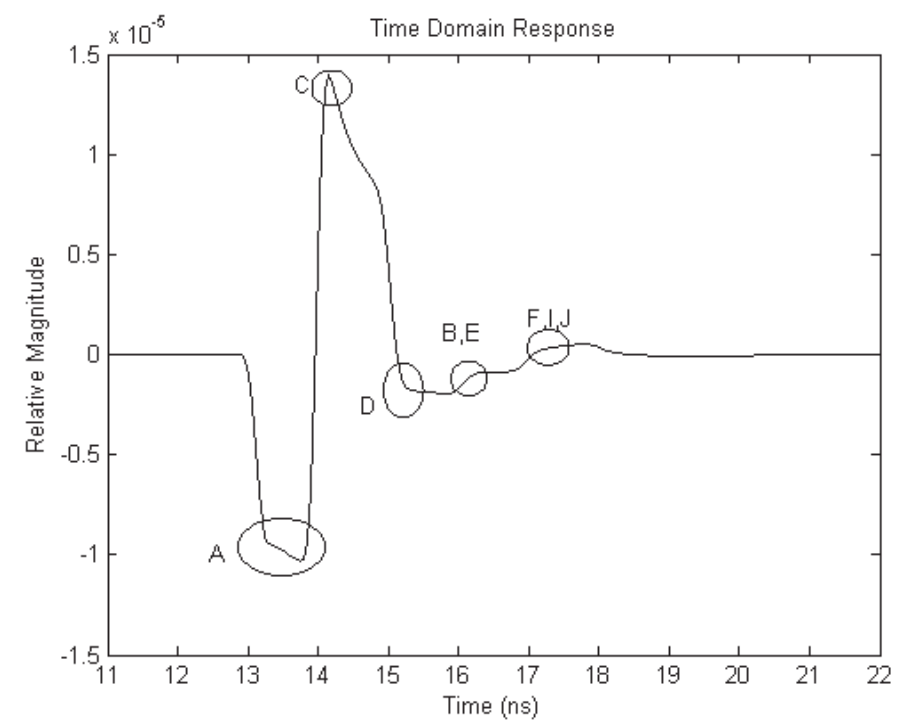

Figure 17c. The time-domain signature for the $14 \mathrm{~cm}$ wire target sited beneath a half-space of Debye tissue at a depth of $14 \mathrm{~cm}$ with an orientation of $\varphi=90^{\circ}$ (horizontal). The time-domain signature was windowed using a Gaussian window. 

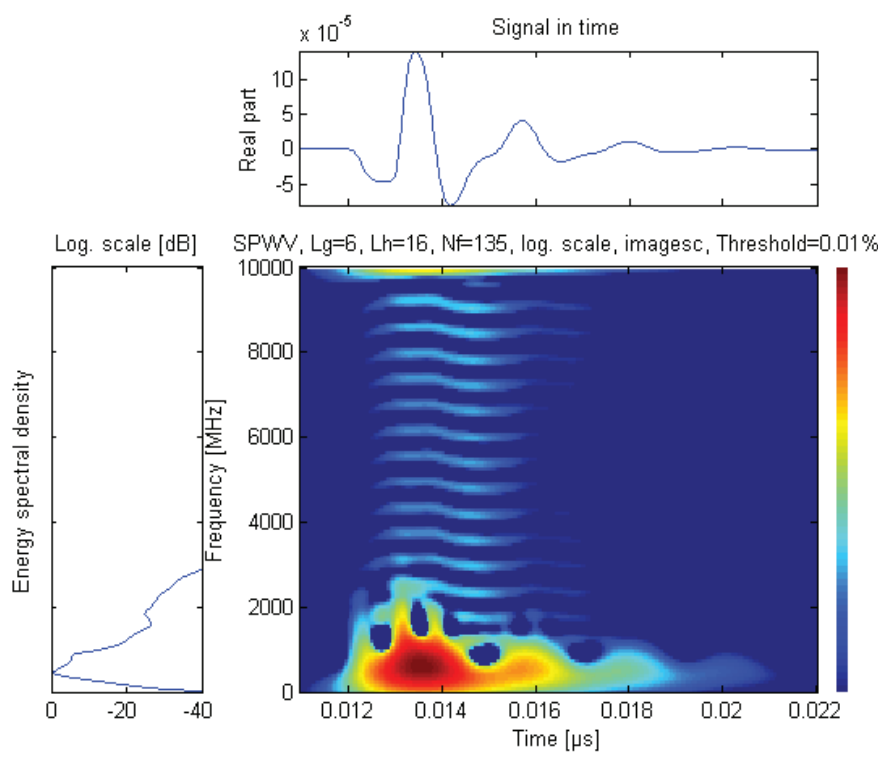

Figure 18a. The smooth pseudo-Wigner-Ville distribution of the target's signature for the $14 \mathrm{~cm}$ wire target sited beneath a half-space of fat-infiltrated tissue at a depth of $14 \mathrm{~cm}$ with an orientation of $\varphi=90^{\circ}$ (horizontal). The timedomain signature was windowed using a Gaussian window.
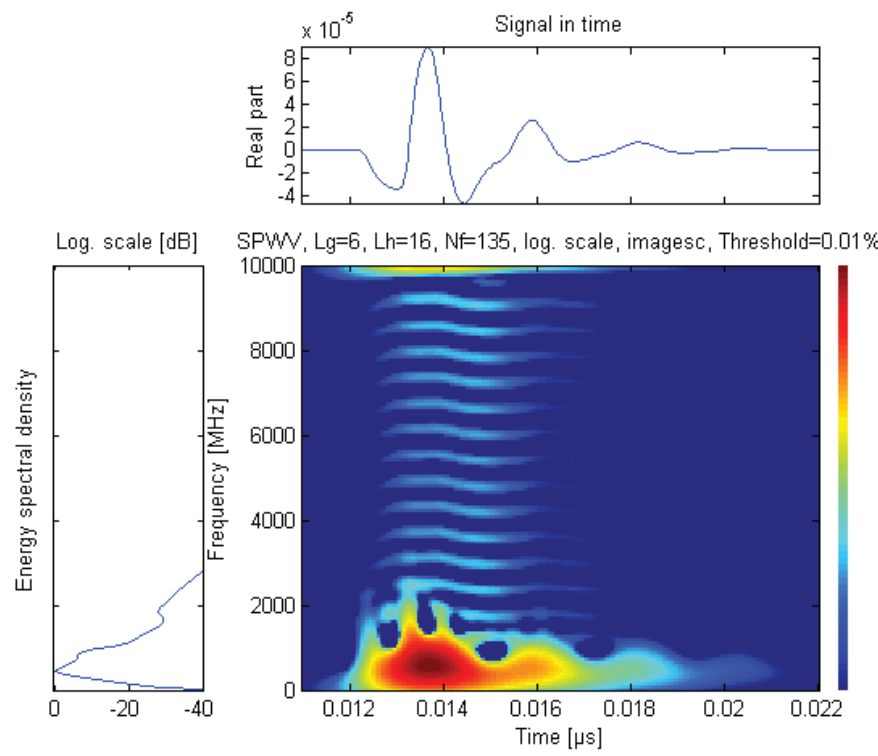

Figure 18b. The smooth pseudo-Wigner-Ville distribution of the target's signature for the $14 \mathrm{~cm}$ wire target sited beneath a layered half-space of fat-infiltrated tissue with a $5 \mathrm{~mm}$ skin layer on top. The wire was sited at a depth of $14 \mathrm{~cm}$ below the air-skin interface with an orientation of $\varphi=90^{\circ}$ (horizontal). The time-domain signature was windowed using a Gaussian window.
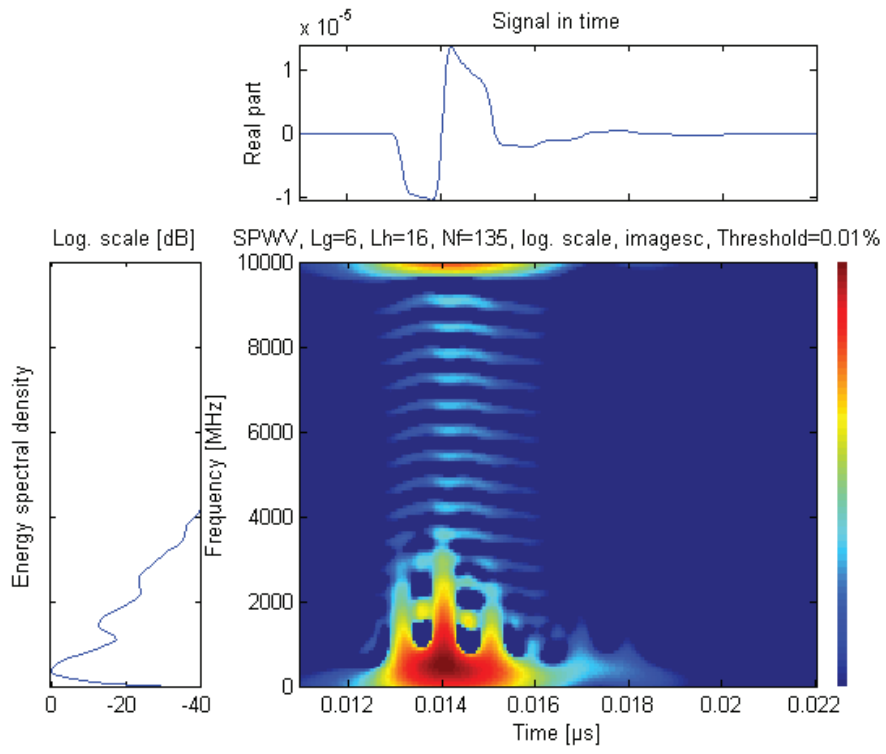

Figure 18c. The smooth pseudo-Wigner-Ville distribution of the target's signature for the $14 \mathrm{~cm}$ wire target sited beneath a half-space of Debye tissue at a depth of $14 \mathrm{~cm}$ with an orientation of $\varphi=90^{\circ}$ (horizontal). The time-domain signature was windowed using a Gaussian window. 


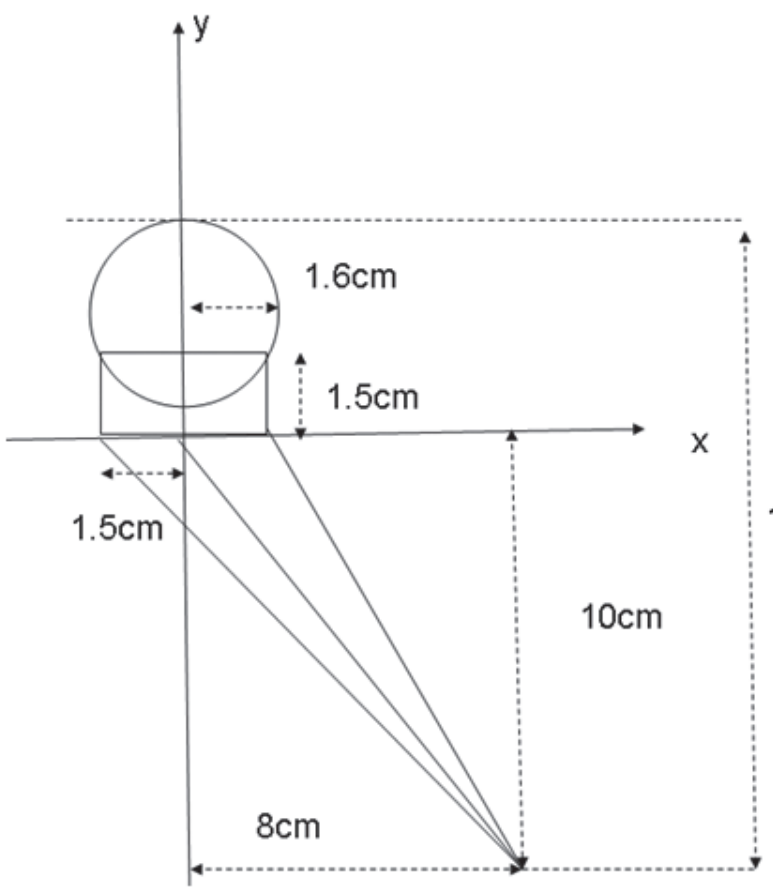

$13.7 \mathrm{~cm}$

Figure 19a. A cross-sectional view of the hip-prosthesis model.

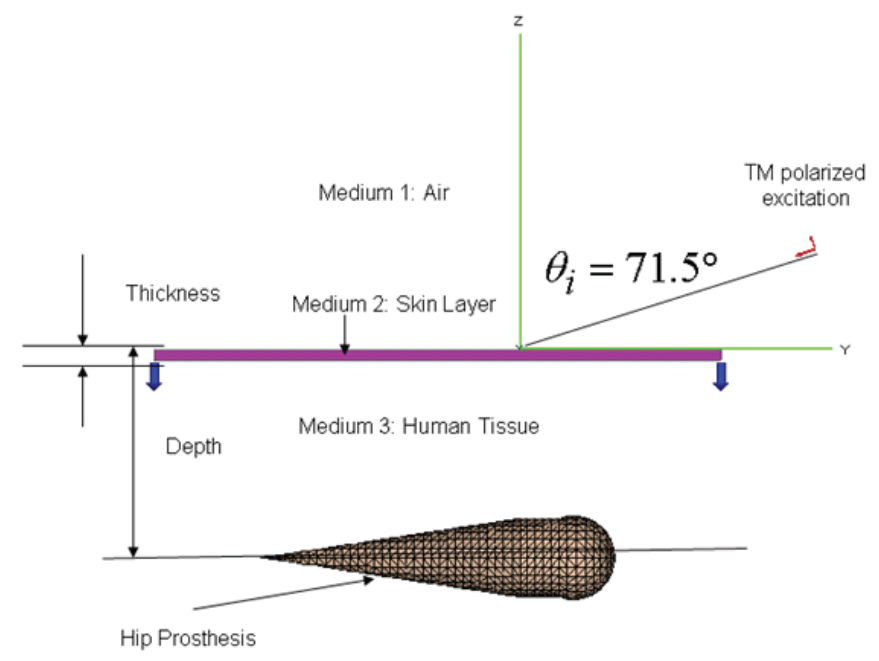

Figure $19 \mathrm{~b}$. The prosthesis under a layered half-space in the FEKO environment.

further investigation. Similar to the previous examples, the nonzero conductivity for a lossy dielectric attenuated the electromagnetic wave, and only the first few dominant scattering phenomena (in the early-time period) in the low-frequency region (where attenuation was less significant) were observed in the time-frequency domain.

\subsection{Examples of a Hip-Prosthesis Model}

In the last example, a hip-prosthesis target sited within a human-tissue model shown in Figure 19 was considered. The objective here was to study the variation of the target's signa- ture as the target's depth varied. Here, only the prosthesis model below a half-space of a Cole-Cole- 4 human-tissue model is given in Figures 16a and 16b. Variations of the properties of the dielectric(s) have been previously addressed for wire targets in the time-frequency domain, and are not repeated.

First, the time-domain target signatures of the prosthesis target at depths of $2.5 \mathrm{~cm}, 8 \mathrm{~cm}$, and $12 \mathrm{~cm}$ below the half-space are shown in Figure 20. It was observed that occurrences of the scattering events were delayed in the time domain as the target depth increased. Furthermore, it was also found that the magnitude of the target's signature decreased with an increase of the target's depth.

The smooth pseudo-Wigner-Ville distributions of the prosthesis at various depths below the human tissue are shown in Figures 21a to 21c for depths of $2.5 \mathrm{~cm}, 8 \mathrm{~cm}$, and $12 \mathrm{~cm}$, respectively. At a depth of $2.5 \mathrm{~cm}$, the first wavefront event appeared as a vertical line at $\sim 11.5 \mathrm{~ns}$ to beyond $7 \mathrm{GHz}$ in Figure 21a. It was also observed that relatively high energy levels were observed in the frequency domain. However, as the target's depth increased, the high-frequency components (above $4 \mathrm{GHz}$ ) suffered more-significant attenuation compared to the low-frequency components (below $4 \mathrm{GHz}$ ). This could be observed in the frequency-domain plots shown in Figure $21 \mathrm{~b}$ and Figure $21 \mathrm{c}$ for $8 \mathrm{~cm}$ and $12 \mathrm{~cm}$ depths, respectively. In the time-frequency domain, it was observed that the high-frequency components mainly contributed to the first wavefront component, and the maximum frequency decreased to $\sim 4.5 \mathrm{GHz}$ and $\sim 4 \mathrm{GHz}$ respectively for $8 \mathrm{~cm}$ and $12 \mathrm{~cm}$ depths (Figures 21b and 21c) .

Compared to the wire target, the prosthesis model had a projected length of $13.7 \mathrm{~cm}$, and thus the fundamental resonant frequencies should have been within similar frequency bands. For both targets, only the first one or two resonant modes below

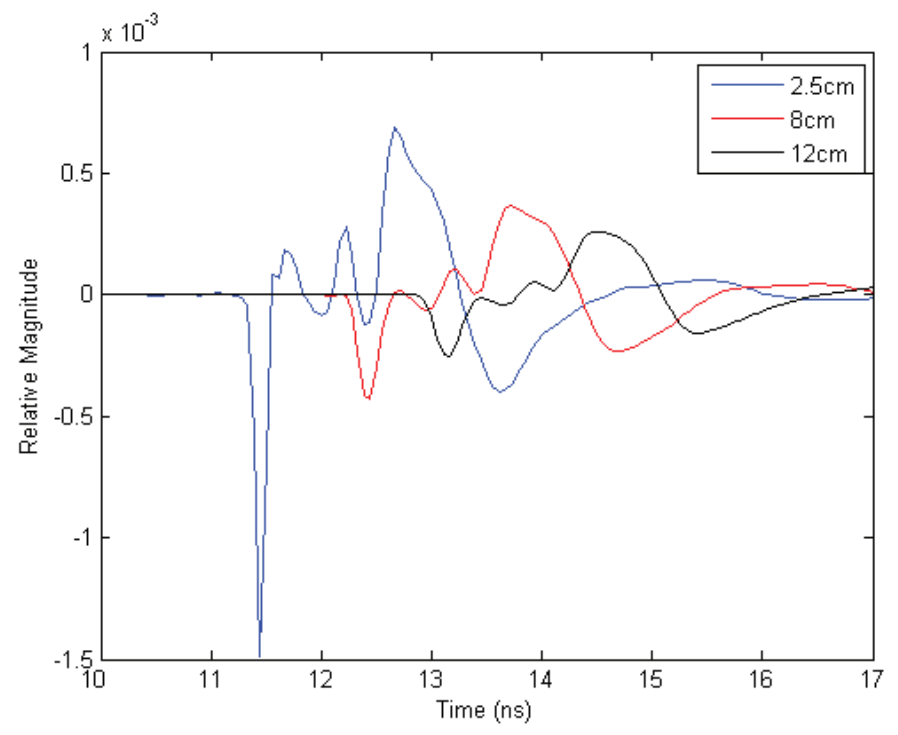

Figure 20. The time-domain signatures for the hip prosthesis sited at depths of $2.5 \mathrm{~cm}, 8 \mathrm{~cm}$, and $12 \mathrm{~cm}$ below a halfspace of fat-infiltrated tissue. The time-domain signatures were windowed using a Gaussian window. 


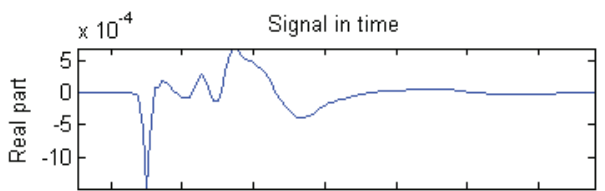

Log. scale [dB] SPW, $L g=6, L h=16, N=135, \log$. scale, imagesc, Threshold $=0.01 \%$
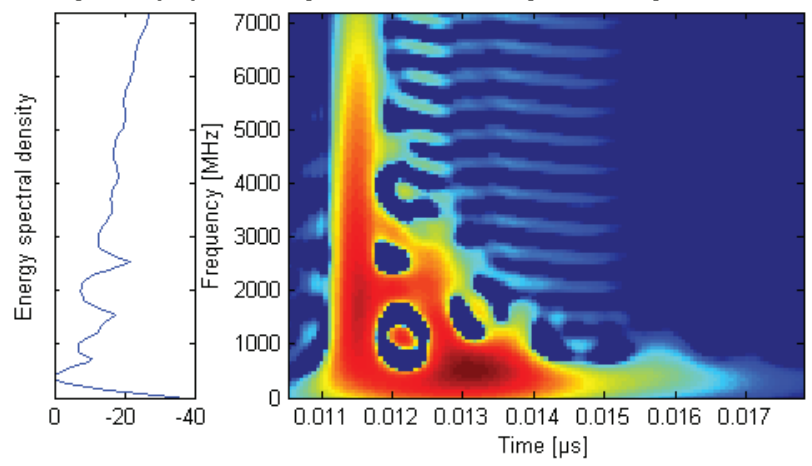

Figure 21a. The smooth pseudo-Wigner-Ville distribution of the target's signature for the hip-prosthesis target sited beneath a half-space of fat-infiltrated tissue at a depth of $2.5 \mathrm{~cm}$. The-time domain signature was windowed using a Gaussian window.
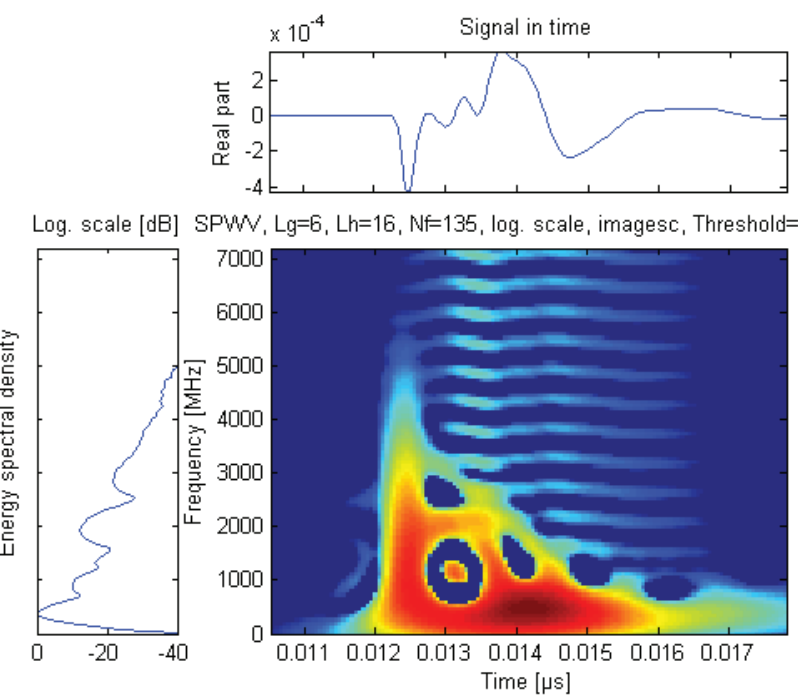

Figure 21b. The smooth pseudo-Wigner-Ville distribution of the target's signature for the hip-prosthesis target sited beneath a half-space of fat-infiltrated tissue at a depth of $8 \mathrm{~cm}$. The-time domain signature was windowed using a Gaussian window.
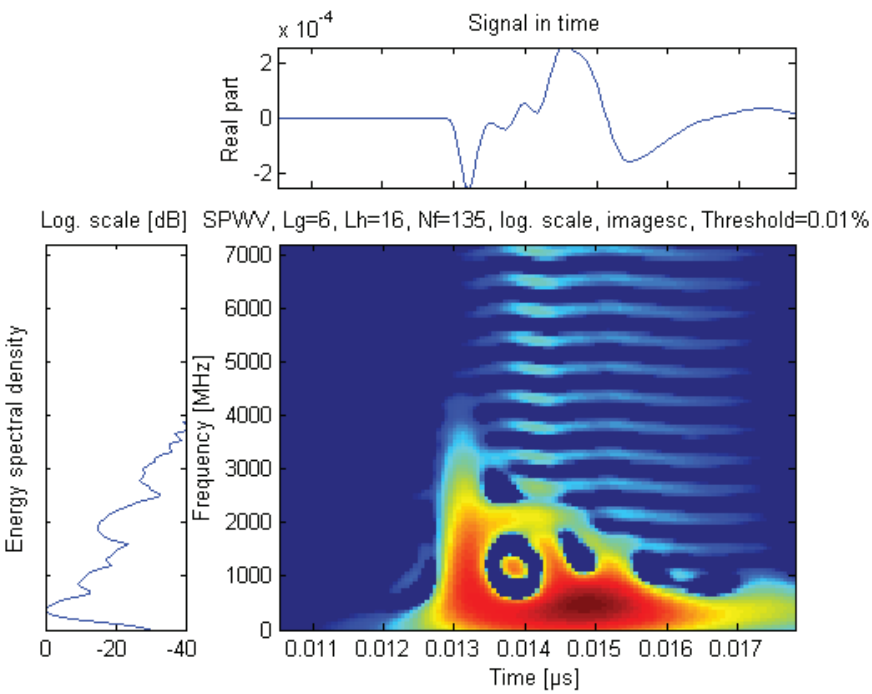

Figure 21c. The smooth pseudo-Wigner-Ville distribution of the target's signature for the hip-prosthesis target sited beneath a half-space of fat-infiltrated tissue at a depth of $12 \mathrm{~cm}$. The-time domain signature was windowed using a Gaussian window. 

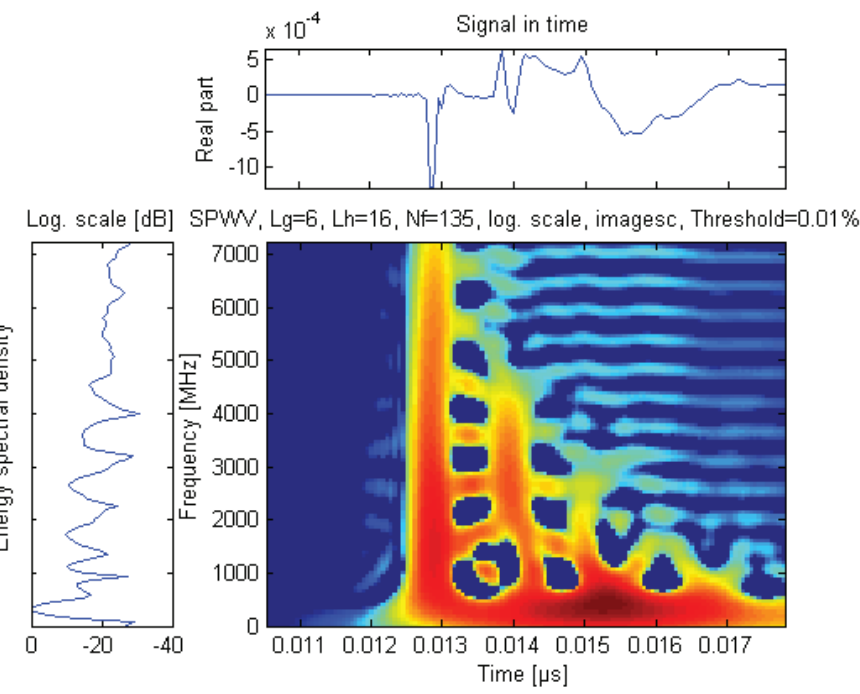

Figure 22. The smooth pseudo-Wigner-Ville distribution of the target's signature for the hip-prosthesis target sited beneath a lossless half-space of $\varepsilon_{r}=9, \sigma=0 \mathrm{~S} / \mathrm{m}$ at a depth of $12 \mathrm{~cm}$. The time-domain signature was windowed using a Gaussian window.

$4 \mathrm{GHz}$ were observed for depths of $14 \mathrm{~cm}$ and $12 \mathrm{~cm}$, respectively, due to the lossy nature of the human tissue. There were only a few wavefront events (vertical lines) observed in the time-frequency domain, and they were the low-order mechanisms that occurred at earlier times. The higher-order scattering mechanisms, due to multiple interactions between the targets and the interfaces, were not observed, as they were significantly attenuated. For the sake of completeness, the timefrequency distributions of the same prosthesis model sited below a lossless half-space of $\varepsilon_{r}=9$ are included in Figure 22 . Compared to the lossy case shown in Figure 20, high-frequency components above $5 \mathrm{GHz}$ were clearly observed in the first two wavefront components, and a number of resonant modes were found above $4 \mathrm{GHz}$. The higher-order multiple interactions were also observed between $15 \mathrm{~ns}$ to $17 \mathrm{~ns}$ in the smooth pseudo-Wigner-Ville distribution shown in Figure 22, below $2 \mathrm{GHz}$.

\section{Contributions Toward Automated Target Recognition}

In this paper, a systematic analysis of transient electromagnetic scattering of metallic targets below homogenous halfspaces was conducted. This started from high- $Q$ targets within a lossless half-space, to targets with lower $Q$ factors within the half-space, and also similar targets within a dispersive lossy half-space. The study showed that higher-order interactions (usually occurring at higher frequencies) were significantly masked by dielectric losses, where present. At the same time, the introduction of a dielectric continuity could also reduce the number of resonant and wavefront events that could be excited. This point has not been well reported in the literature. This implies that for subsurface target recognition, emphasis should be put on robustly exciting the fundamental resonant frequency that corresponds to the global resonant behavior of the entire target. In our recent work concerning a novel subsurface target-recognition method [13], it was shown that better targetrecognition performance could be obtained when only the fundamental resonant mode (corresponding to the projected length of the prosthesis model) was considered in the targetrecognition process, instead of considering all the resonant modes. This again emphasizes the significance and dominance of the first resonant mode at the lower-frequency region in the automatic target-recognition scenario.

At the same time, the substructure [49] or partial [30] resonant modes, which correspond to the resonant behaviors of target substructures, usually have higher resonant frequencies than the fundamental resonant modes. Just as for the higher-order resonances that correspond to the higher-order interactions, these substructure resonances are not well excited when the target is sited within a lossy environment.

To probe further, consider an automatic target-recognition scenario consisting of two similar targets with small geometrical differences that can only be revealed via substructure resonances [49]. If the two targets are in free space, successful target recognition can be done via a "banded" E-pulse technique [50], which aims to differentiate the targets based on their substructure resonances. However, if they are both sited in a lossy half-space, their global resonances could be very similar. According to the above findings, it is very likely that their substructure and higher-order resonances are not well excited, and resonance-based target recognition may fail. Under such circumstances, one may need to incorporate other feature sets, such as characteristic polarization states (CPS) together with their global resonant frequencies [51].

\section{Conclusions}

A series of examples using time-frequency analysis for a PEC wire target and the more-complicated hip-prosthesis model, below various half-spaces, have been presented. Targets embedded in both lossless and lossy half-spaces, with different dielectric properties, target orientations, and target depths, have all been studied. Using time-frequency analysis, it was found that at some orientations, the wavefront and resonance behaviors interacted with each other, while some did not. Higher-order scattering, based on multiple interactions between the target and the interfaces, were clearly observed when the target was sited within a lossless half-space. However, as the half-space became lossy, attenuation was introduced, and higher-order scattering became less obvious, especially when the target depth was increased. In addition, the signal was smeared, due to the dispersive nature of the half-space.

It is worth noting that in some cases, the wavefront and resonance behaviors interacted with each other. This was similar to the findings in [30] for a target in free space, and can be explained by the Hybrid Wavefront Singularity Expansion Method model. With the introduction of an air-medium 
interface, some of the wavefront and resonant events were not well excited. Furthermore, positive and negative slopes occasionally appeared in the time-frequency domain, and this was probably due to the dispersive nature of the half-space. Again, this requires further study.

The examples presented were all PEC targets. For landmines and other applications such as ground-penetrating radar, the target is usually a relatively low- $Q$ (dielectric) target. The wavefront and resonance behaviors are thus not well observed in the time-frequency domain, such as examples given in [31, 32]. However, it would be interesting to study the transientscattering behavior of penetrable dielectric targets (with the inclusion of internal resonances) inside in the lossless dielectric medium, or sited below a half-space [52], using time-frequency analysis. Compared to the non-penetrable PEC target, the scattering behavior is much more complicated, as the dielectric contrast between the background medium and object plays an important role.

Lastly, it is important to see that the findings here are closely related to some observations from our recently developed automatic target-recognition schemes [13, 50]. There could be limitations for resonance-based target recognition for subsurface target recognition of targets with sub-structural differences. Further investigations are thus required to look into novel target-recognition algorithms to handle such cases, for instance [51].

\section{Acknowledgement}

This work was supported in part by the Australian Research Council (ARC), under Grant DP0557169.

\section{References}

1. P. Ilavarasan, J. E. Ross, E. J. Rothwell, K. M. Chen, D. P. Nyquist, "Performance of an Automated Radar Target Pulse Discrimination Scheme Using E pulses and S Pulses," IEEE Transactions on Antennas and Propagation, AP-41, 5, May 1993, pp. 582-588.

2. K. M. Chen, D. P. Nyquist, E. J. Rothwell, L. L. Webb, B. Drachman, "Radar Target Discrimination by Convolution of Radar Return with Extinction-Pulses and Single-Mode Extraction Signals," IEEE Transactions on Antennas and Propagation, AP-34, 7, July 1986, pp. 896-904.

3. C. E. Baum, E. J. Rothwell, K. M. Chen, D. P. Nyquist, "The Singularity Expansion Method and Its Application to Target Identification," Proceedings of the IEEE, 79, 10, October 1991, pp. 1481-1491.

4. C. E. Baum, "The Singularity Expansion Method" in L. B. Felsen (ed.), Transient Electromagnetic Fields, New York, Springer-Verlag, 1976, pp. 129-179.
5. E. M. Kennaugh, "The K-Pulse Concept," IEEE Transactions on Antennas and Propagation, AP-29, 2, March 1981, pp. 327-331.

6. E. Rothwell, D. P. Nyquist, K. M. Chen, B. Drachman, "Radar Target Discrimination using the Extinction-Pulse Technique," IEEE Transactions on Antennas and Propagation, AP-33, 9, September 1985, pp. 929-936.

7. S. Vitebskiy and L. Carin, "Resonances of Perfectly Conducting Wires and Bodies of Revolution Buried in a lossy Dispersive Half-Space," IEEE Transactions on Antennas and Propagation, AP-44, 12, December 1996, pp. 1575-1583.

8. N. Geng, D. R. Jackson, L. Carin, "On the Resonances of a Dielectric BOR Buried in a Dispersive Layered Medium," IEEE Transactions on Antennas and Propagation, AP-47, 8, August 1999, pp. 1305-1313.

9. Y. Wang, I. D. Longstaff, C. J. Leat, N. V. Shuley, “Complex Natural Resonances of Conducting Planar Objects Buried in a Dielectric Half-Space," IEEE Transactions on Geoscience and Remote Sensing, 39, 6, June 2001, pp. 1183-1189.

10. Y. Wang, On the Characteristics of Small Buried Objects Illuminated with UWB Radar, $\mathrm{PhD}$ dissertation, Cooperative Research Center for Sensor Signal and Information Processing, School of Computer Science and Electrical Engineering, The University of Queensland, 2000.

11. H. S. Lui and N. V. Z. Shuley, "Detection of Depth Changes of a Metallic Target Buried Inside a Lossy Halfspace Using the E-Pulse Technique," IEEE Transactions on Electromagnetic Compatibility, 49, 4, November 2007, pp. 868-875.

12. H. S. Lui, F. Aldhubaib, N. V. Z. Shuley, H. T. Hui, "Subsurface Target Recognition Based on Transient Electromagnetic Scattering," IEEE Transactions on Antennas and Propagation, AP-57, 10, October 2009, pp. 3398-3401.

13. H. S. Lui, N. V. Z. Shuley, A. D. Rakic, "A Novel, Fast, Approximate Target Detection Technique for Metallic Target Below a Frequency Dependant Lossy Halfspace," IEEE Transactions on Antennas and Propagation, AP-58, 5, May 2010, pp. 1699-1710.

14. C. E. Baum, "Representation of Surface Current Density and Far Scattering in EEM and SEM With Entire Functions," in P. P. Delsanto and A. W. Saenz, New Perspectives on Problems in Classical and Quantum Physics, Part II: Acoustic Propagation and Scattering, Electromagnetic Scattering, London, Gordon and Breach, 1998, pp. 273-316.

15. M. A. Richards," SEM Representations of the Early and Late Time Fields Scattered from Wire Targets," IEEE Transactions on Antennas and Propagation, AP-42, 4, pp. 564-566, April 1994. 
16. E. Heyman and L. B. Felsen, "A Wavefront Interpretation of the Singularity Expansion Method," IEEE Transactions on Antennas and Propagation, AP-33, 7, July 1985, pp. 706-718.

17. L. B. Felsen, "Progressing and Oscillatory Waves for Hybrid Synthesis of Source Excited Propagation and Diffraction," IEEE Transactions on Antennas and Propagation, AP32, 8, August 1984, pp. 775-796.

18. H. Shirai and L. Felsen, "Modified GTD for Generating Complex Resonances for Flat Strips and Disks," IEEE Transactions on Antennas and Propagation, AP-34, 10, October 1986, pp. 779-790.

19. H. Shirai and L. Felsen, "Wavefront and Resonance Analysis of Scattering by a Perfectly Conducting Flat Strip," IEEE Transactions on Antennas and Propagation, AP-34, 10, October 1986, pp. 1196-1207.

20. H. Shirai, "De-Emphasizing Low Frequency Defects in GTD Analysis of Pulsed Signal Scattering by a Perfectly Conducting Flat Strip," IEEE Transactions on Antennas and Propagation, AP-34, 10, October 1986, pp. 1261-1266.

21. V. C. Chen and H. Ling, Time-Frequency Transforms for Radar Imaging and Signal Analysis, Norwood, MA, Artech House, 2002.

22. H. Kim and H. Ling, "Wavelet Analysis of Electromagnetic Backscatter Data," Electronics Letters, 28, 3, January 1992, pp. 279-281.

23. J. Moore and H. Ling, "Time-Frequency Analysis of the Scattering Phenomenology in Finite Dielectric Gratings," Microwave and Optical Technology Letters, 6, 10, 1993, pp. 597-600.

24. H. Kim and H. Ling, "Wavelet Analysis of Radar Echo from Finite-Size Targets," IEEE Transactions on Antennas and Propagation, AP-41, 2, February 1993, pp. 200-207.

25. H. Ling, J. Moore, D. Bouche, V. Saavedra, "Time-Frequency Analysis of Backscattered Data from a Coated Strip with a Gap," IEEE Transactions on Antennas and Propagation, AP-41, 8, August 1993, pp. 1147-1150.

26. C. Ozdemir and H. Ling, "Joint Time-Frequency Interpretation of Scattering Phenomenology in Dielectric-Coated Wires," IEEE Transactions on Antennas and Propagation, AP45, 8, August, 1997, pp. 1259-1264.

27. V. C. Chen and H. Ling, "Joint Time-Frequency Analysis for Radar Signal and Image Processing," IEEE Signal Processing Magazine, 16, 2, March 1999, pp. 81-93.

28. H. Ling and H. Kim, "Wavelet Analysis of Backscattering Data from an Open-Ended Waveguide Cavity," IEEE Microwave and Wireless Components Letters, 2, 4, April 1992, pp. 140-142.
29. E. J. Rothwell, K. M. Chen, D. P. Nyquist, “An Adaptive Window Width Short Time Fourier Transform for Visualization of Radar Target Substructure Resonances," IEEE Transactions on Antennas and Propagation, AP-46, 9, September 1998, pp. 1393-1395.

30. H. S. Lui and N. V. Z. Shuley, "Evolutions of Partial and Global Resonances in Transient Electromagnetic Scattering," IEEE Antennas and Propagations Letters, 7, 2008, pp. 436-439.

31. G. C. Gaunaurd and L. H. Nguyen, "Detection of LandMines Using Ultra Wideband Radar Data and Time-Frequency Signal Analysis," IEE Proceedings - Radar, Sonar and Navigation, 151, 5, October 2004, pp. 307-316.

32. T. G. Savelyev, L. van Kempen, H. Sahli, J. Sachs, and M. Sato, "Investigation of Time-Frequency Features for GPR Landmine Discrimination," IEEE Transactions on Geoscience and Remote Sensing, 45, 1, January 2007, pp. 118-129.

33. S. Vitebskiy and L. Carin, "Moment-Method Modeling of Short-Pulse Scattering from and the Resonances of a Wire Buried Inside a Lossy, Dispersive Half-Space," IEEE Transactions on Antennas and Propagation, AP-43, 11, November 1995, pp. 1303-1312.

34. T. Dogaru and L. Carin, "Time-Domain Sensing of Targets Buried Under a Rough Air-Ground Interface," IEEE Transactions on Antennas and Propagation, AP-46, 3, March 1998, pp. 360-372.

35. S. Vitebskiy, K. Sturgess, L. Carin, "Short-Pulse PlaneWave Scattering from Buried Perfectly Conducting Bodies of Revolution," IEEE Transactions on Antennas and Propagation, AP-44, 2, February 1996, pp. 153-1151.

36. O. Rioul and P. Flandrin, "Time-Scale Energy Distributions: A General Class Extending Wavelet Transforms," IEEE Transactions on Signal Processing, 40, 7, July 1992, pp. 17461757.

37. F. Auger and P. Flandrin, "Improving the Readability of Time-Frequency and Time-Scale Representations by the Reassignment Method," IEEE Transactions on Signal Processing, 43, 5, May 1995, pp. 1068-1089.

38. J. Jeong and W. J. Williams, "Kernel Design for Reduced Interference Distributions," IEEE Transactions on Signal Processing, 40, 2, February 1992, pp. 402-412.

39. F. Hlawatsch and G. F. Boudreaux-Bartels, "Linear and Quadratic Time-Frequency Signal Representations," IEEE Signal Processing Magazine, 9, 2, pp. 21-67, April 1992.

40. L. Cohen, Time-Frequency Analysis, Englewood Cliffs, NJ, Prentice Hall PTR, 1995.

41. F. Auger, P. Flandrin, P. Goncalves, and O. Lemoine, "TimeFrequency Toolbox - For Use with MATLAB," CNRS (France) and Rice University (USA), 1996. 
42. G. C. Gaunaurd and H. C. Strifors, "Signal Analysis by Means of Time-Frequency (Wigner-Type) DistributionsApplications to Sonar and Radar Echoes," Proceedings of the IEEE, 84, 9, September 1996, pp. 1231-1248.

43. H. S. Lui and N. Shuley "On the Analysis of Electromagnetic Transients from Radar Targets using Smooth Pseudo Wigner-Ville Distribution (SPWVD)," IEEE International Symposium on Antennas and Propagation Digest, Honolulu, Hawaii, USA, June 10-15, 2007, pp. 5701-5704.

44. S. Gabriel, R. W. Lau, C. Gabriel, "The Dielectric Properties of Biological Tissues: III. Parametric Models for the Dielectric Spectrum of Tissues," Physics in Medicine and Biology, 41, 11, November 1996, pp. 2271-2293.

45. Y. Huo, Rajeev Bansal, Q. Zhu, "Modeling of Noninvasive Microwave Characterization of Breast Tumors," IEEE Transactions on Biomedical Engineering, 51, 7, July 2004, pp. 1089-1094.

46. C. A. Balanis, Advanced Engineering Electromagnetics, New York, John Wiley \& Sons, 1989.

47. FEKO EM Software \& Systems S.A., (Pty) Ltd., 32 Techno Lane, Technopark, Stellenbosch, 7600, South Africa.

48. S. M. Rao, Time Domain Electromagnetics, San Diego, Academic Press, 1999.

49. C. E. Baum, "Substructure SEM," F. Sabath et al., (eds.), Ultra-Wideband Short-Pulse Electromagnetics 7, New York, Springer, 2007, Chapter 72, pp. 681-689.

50. H. S. Lui and N. V. Z. Shuley, "Radar Target Identification Using a 'Banded' E-Pulse Technique," IEEE Transactions on Antennas and Propagation, AP-54, 12, December 2006, pp. 3874-3881.

51. F. F. H. Aldhubaib and N. V. Z. Shuley, "Characteristic Polarization States Estimation in an Ultrawideband Context: A Frequency Domain Approach," IEEE Transactions on Geoscience and Remote Sensing, 47, 8, August 2009, pp. 28082817.

52. C. -C. Chen, "Electromagnetic Resonances of Immersed Dielectric Spheres," IEEE Transactions on Antennas and Propagation, AP-46, 7, July 1998, pp. 1074-1083.

\section{Introducing the Feature Article Authors}

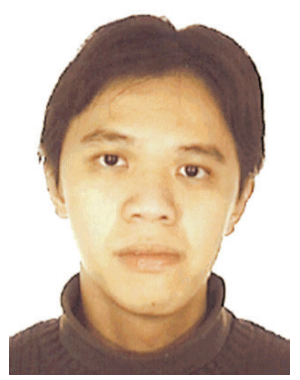

Hoi-Shun Lui received his Bachelor of Engineering degree with first class honors and his $\mathrm{PhD}$ degree in Electrical Engineering from the University of Queensland, Brisbane, Australia in 2003 and 2008, respectively. During his candidature, he carried out consultancy work with Filtronics Australia Ltd. In 2008, he was a Research Fellow with the Microwave and RF Group at the National University of Singapore. He is now an Assistant Professor with the Department of Signals and Systems at Chalmers University of Technology, Gothenburg, Sweden. He also serves as a project leader in the VINN Excellence centre CHASE, which is financed by the Swedish Governmental Agency for Innovation Systems (VINNOVA), industry, and Chalmers.

Dr. Lui is an active reviewer in various international refereed journals, such as the IEEE Transactions on Antennas and Propagation and the IEEE Antennas and Wireless Propagation Letters. His research interests include resonance-based radartarget recognition, transient electromagnetic scattering, mutual coupling of antenna arrays, and direction-of-arrival estimation.

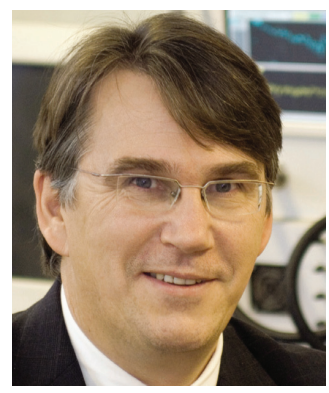

Mikael Persson received his $\mathrm{MSc}$ and $\mathrm{PhD}$ from Chalmers University of Technology, Gothenburg, Sweden, in 1982 and 1987, respectively. In 2000, he became Professor in Electromagnetics, and in 2006, Professor in Biomedical Electromagnetics, at the Department of Signals and Systems, at 
Chalmers University of Technology. He is presently the head of the Division of Signal Processing and Biomedical Engineering, and Director of the regional research and development platform, MedTech West. At present, these activities involve approximately 50 researchers. His main research interests include electromagnetic diagnostics and treatment. $\mathrm{He}$ is the author/coauthor of more than 200 refereed journal and conference papers.

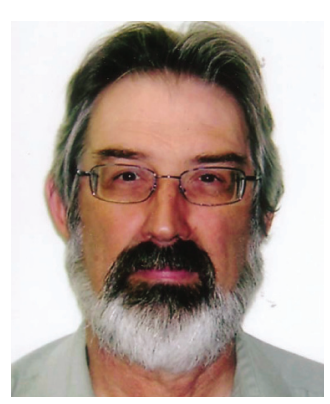

Nicholas V. Z. Shuley received the BE and MEngSc from the University of New South Wales, Australia, in 1973 and 1975, respectively, and the $\mathrm{PhD}$, again in Electrical Engineering, from Chalmers University of Technology, Gothenburg, Sweden, in 1985.

From 1977 to 1978 , he was with Microwave Associates, Dunstable, UK. From 1979 to 1988, he was a Research and Teaching Assistant and later a Post-Doctoral Scientist with the Division of Network Theory at Chalmers University of Technology in Gothenburg, Sweden. From 1988 to 1998, he was with the University of Queensland, working within the Microwave Group. During 1996, he was awarded a Visiting Scientist Stipend by the Spanish Government to work on electromagnetic-related problems at the Department of Electronics and Electromagnetics within the Department of Physics at the University of Seville, Spain. From 1999 to 2001, he was Head of the Electronics discipline within the School of Electrical and Computer Systems Engineering at RMIT University in Melbourne, Australia. He returned in 2002 to the University of Queensland to the School of Information Technology and Electrical Engineering, where he is currently teaching and supervising research in areas relating to time-domain electromagnetics and non-cooperative object identification.

Dr. Shuley has carried out consulting work for the European Space Agency, has been a member of the Editorial Board of the IEEE Microwave Theory and Techniques Society since 1992, and shared a Best Paper Award at JINA 1988. He has extensively published in both international journals and conference proceedings concerned with electromagnetic phenomena, and has successfully supervised (jointly or solely) 18 masters or $\mathrm{PhD}$ students to completion.

\section{Addendum}

Since the article by Krishnasamy T. Selvan, "Fundamentals of Electromagnetic Units and Constants" (IEEE Antennas and Propagation Magazine, 54, 3, June 2012, pp. 100-114) appeared in print, two relevant previous papers $[1,2]$ have come to the author's attention. Leo Young's article [1] presents an interesting discussion, with examples, of conversion and comparison between different systems of electromagnetic units. The more recent article by Pelosi and Sellari [2] provides a historical account of the measurement and naming of the velocity of light, $c$.

\section{References}

1. L. Young, "Electromagnetic Units and Equations," IEEE Transactions on Microwave Theory and Techniques, 50, 3, March 2002, pp. 1021-1027.

2. G. Pelosi and S. Selleri, "Does ' $c$ ' stand for speed or constancy?" IEEE Antennas and Propagation Magazine, 52, 6, December 2010, pp. 207-219.

Krishnasamy T. Selvan

E-mail: ktselvan@ieee.org 